\title{
https://helda.helsinki.fi
}

\section{High levels of auxin signalling define the stem-cell organizer of the vascular cambium}

\section{Smetana, Ondrej}

2019-01-24

Smetana , O , Mäkilä , R , Lyu , M , Amiryousefi , A , Rodriguez , F S , Wu , M-F , Sole-Gil , A , Gavarron , M L , Siligato , R , Miyashima , S , Roszak , P , Blomster , T , Reed , J W , Broholm , S \& Mähönen , A P 2019 , ' High levels of auxin signalling define the stem-cell organizer of the vascular cambium ' , Nature , vol. 565 , no. 7740 , pp. 485-+ . https://doi.org/10.1038/s41586-018-08

http://hdl.handle.net/10138/325525

https://doi.org/10.1038/s41586-018-0837-0

acceptedVersion

Downloaded from Helda, University of Helsinki institutional repository.

This is an electronic reprint of the original article.

This reprint may differ from the original in pagination and typographic detail.

Please cite the original version. 


\section{High auxin signalling defines stem cell organiser of the vascular cambium}

2

3

Ondřej Smetana ${ }^{1,2}$, Riikka Mäkilä ${ }^{1,2}$, Munan Lyu ${ }^{1,2}$, Ali Amiryousefi' ${ }^{2}$, Filomeno Sánchez Rodríguez ${ }^{1,2}$, Miin-Feng $\mathrm{Wu}^{3}$, Anna Solé Gil ${ }^{1,2}$, Marina Leal Gavarrón ${ }^{1,2}$, Riccardo Siligato ${ }^{1,2}$, Shunsuke Miyashima ${ }^{1,2}$, Pawel Roszak ${ }^{1,2}$, Tiina Blomster ${ }^{1,2}$, Jason W. Reed ${ }^{3}$, Suvi Broholm ${ }^{1,2}$ \& Ari Pekka Mähönen ${ }^{1,2, *}$

1. Institute of Biotechnology, HiLIFE, University of Helsinki, Helsinki 00014, Finland

2. Organismal and Evolutionary Biology Research Programme, Faculty of Biological and Environmental Sciences, and Viikki Plant Science Centre, University of Helsinki, Helsinki 00014, Finland

3. Department of Biology, University of North Carolina at Chapel Hill, U.S.A

* Author for correspondence: AriPekka.Mahonen@helsinki.fi and phloem outside of the cambium ${ }^{1}$. Morphogenesis in plants is typically coordinated by organiser cells which direct the adjacent stem cells to undergo programmed cell division and differentiation. It is unknown where the vascular cambium stem cells are located and whether the organiser concept applies to the cambium². Here, we combine lineage tracing and molecular genetic studies in Arabidopsis thaliana roots to show that cells with xylem identity direct adjacent vascular cambial cells to divide and function as stem cells. Thus, these xylem identity cells constitute an organiser. A local maximum of the phytohormone auxin and consequent expression of class III homeodomain-leucine zipper (HD-ZIP III) transcription factors promote xylem identity and cellular quiescence of the organiser cells. Additionally, the organiser maintains phloem identity cell nonautonomously. Consistent with this dual function of the organiser cells, xylem and phloem originate from a single, bifacial stem cell in each radial cell file, thus confirming the classical theory of a uniseriate vascular cambium $^{3}$. Ectopically activated high auxin signalling clones differentiate as xylem vessels, and induce cell divisions and expression of cambial and phloem markers in the adjacent cells, suggesting that local auxin signalling maximum is sufficient to specify a stem cell organiser. Although vascular cambium has a unique function among plant meristems, its stem cell organiser shares features with the root and shoot meristem organisers. 
Plant organs grow axially during primary development, and then radially during secondary development. In many plant organs, including woody stems, secondary development is organised by two concentric, cylindrical meristems undergoing periclinal cell divisions (i.e., the division plane is parallel to the surface of an organ or a tissue): the inner vascular cambium proliferates to produce conductive xylem and phloem, and the outer phellogen (also called cork cambium) produces protective tissue called phellem ${ }^{4}$. In the Arabidopsis thaliana root, secondary meristems originate from parenchymal cells located between primary xylem and phloem, known as procambium, and from cells surrounding the procambium, xylem and phloem, known as the pericycle ${ }^{5,6}$ (Fig. 1a, Extended Data Fig. 1a, b).

In order to understand at cellular resolution how the vascular cambium and phellogen develop from the procambium and pericycle, we designed a novel CRE/lox recombination system to carry out cell lineage tracing in the Arabidopsis root (Extended Data Fig. 2a, b). Clones marked with $\beta$-Glucuronidase (GUS) expression were induced during the activation of root secondary growth (Fig. 1a, b), and the subsequent sectors were analysed after 11 days. GUS sectors showed highly repetitive patterns indicating that the secondary development is a tightly controlled process (Fig. 1b, Extended Data Fig. 2c). This enabled us to develop a fate map of procambial and pericycle cells during the morphogenesis of root secondary tissue (Fig. 1b). The fate map showed that phellogen originates from the pericycle (dark and light blue, in Fig. 1b), confirming previous anatomical observations $^{4-6}$. Pericycle cells adjacent to the primary xylem (xylem pole pericycle (XPP) cells, dark blue in Fig. 1b) are also founder cells of the vascular cambium. XPP cell proliferation thus leads to separation of the two concentric meristems and production of all the secondary tissues from their circumferential position (Extended Data Fig. 2d). Our finding underscores the pluripotency of XPP cells, which are also the source of lateral roots and regenerated tissue (callus) ${ }^{7}$. The fate map also revealed that only those procambial cells that are in physical contact with primary xylem vessels (red, in Fig. 1b) give rise to vascular cambium, and secondary xylem and phloem. Thus, these xylem-adjacent procambial cells together with xylem-adjacent daughters of XPP cells act as the stem cells of vascular cambium during the activation of secondary growth. The fact that procambial and pericycle cells that are not in contact with xylem (pink and light blue, respectively in Fig. 1b) do not form vascular cambium suggests that cellular position (adjacent to xylem) rather than identity during primary development determines the origin of the vascular cambium. This conclusion is further supported by lineage tracing in roots transiently treated with the phytohormone cytokinin, which gives cells in protoxylem position procambium identity. Located adjacent to the remaining xylem, these cells formed only vascular cambium, while cells in the XPP position, no longer adjacent to xylem cells, formed only phellogen and not vascular cambium (Extended Data Fig. $2 \mathrm{~d}-\mathrm{g}$ ). Together, these findings suggest that xylem or xylem precursor cells cause neighbouring cells to become vascular cambium stem cells. 
At the onset of secondary growth (Fig. 1a), primary xylem cells have already undergone programmed cell death as the final step of their differentiation. We therefore hypothesize that xylem induces stem cell identity in the neighbouring cells earlier, during primary development, when cells with xylem identity are still alive and capable of signal transduction. A family of five redundantly acting HD-ZIP III transcription factors determine xylem identity during the primary development of the Arabidopsis root ${ }^{8-10}$. Additionally, an HD-ZIP III orthologue in Populus regulates vascular cambium morphogenesis ${ }^{11}$. HD-ZIP IIIs are thus likely candidates for regulating signals for stem cell identity. HD-ZIP IIIs are expressed in the cambial zone, and at least AtHB8 is expressed predominantly on the xylem side of the vascular cambium, adjacent to the cells undergoing cell division (highlighted with AINTEGUMENTA (ANT) expression), thus consistent with the idea that they mark cells with secondary xylem identity (Fig. 2a, Extended Data Fig. 3a, b). Only mutants lacking at least four of the genes show patterning defects and reduced xylem formation in the primary root vasculature ${ }^{8}$. The same mutant combinations also show scattered cambial divisions and xylem patterning during secondary development (Extended Data Fig. 3c, d). To examine whether these severe developmental defects are a direct consequence of the loss of HD-ZIP IIIs or a secondary consequence of defects in primary development, we constructed an inducible ${ }^{12}$ miR165a line that targets the mRNAs of all five HD-ZIP IIIs ${ }^{13}$ (Extended Data Fig. 4a). Ubiquitous induction of miR165a from germination onwards severely inhibited primary xylem differentiation (Extended Data Fig. 4b), similar to the quintuple HD-ZIP III mutant ${ }^{8}$. Induction of miR165a during the activation of secondary growth initially blocked vessel differentiation, indicating that the HD-ZIP IIIs are also required for secondary xylem vessel formation (Fig. 2b). Dispersed cell divisions and erratic xylem vessel formation occurred in the remaining undifferentiated tissue, resembling the phenotypes of quadruple HD-ZIP III mutants (Extended Data Fig. 3d). Induction of miR165a during secondary growth forced xylem parenchyma cells and cells with xylem identity, which were previously quiescent, to re-enter mitosis, generating a population of dividing cells in the place of xylem (Fig. 2c, Extended Data Fig. 4c). These results indicate that the HD-ZIP IIIs are required for cellular quiescence in xylem cells. WUSCHEL-RELATED HOMEOBOX $4(\text { WOX4) })^{14}$ and PHLOEM INTERCALATED WITH XYLEM/TDIF RECEPTOR (PXY/TDR $)^{14,15}$ are key cambial regulators. They show expression maxima on the xylemside of the cambium and weak expression in the adjacent dividing cells (marked with ANT expression), thus strongly resembling the AtHB8 expression pattern (Fig. 2a). Induction of miR165a led to downregulation of WOX4 and PXY (Fig. 2d, Extended Data Fig. 4d). These data imply that xylem identity regulators, the HD-ZIP IIIs, define a stem cell organiser which cell non-autonomously confers cambium stem cell identity to the adjacent cells.

Unexpectedly, loss of the HD-ZIP IIIs also caused decreased production of differentiated phloem cells (Extended Data Fig. 4e) and lower expression of early phloem marker PHLOEM-EARLY-DOF 1 (PEAR1) 16, 17 (Fig. 2a, d, Extended Data Figs 1a, 4d), suggesting that the organiser cells maintain a common stem cell for secondary 
xylem and phloem. To test this hypothesis, we traced cell lineages during secondary growth. GUS clones were generated in 15-day or 30-day old Arabidopsis roots, which contain 2-4 (15-day) or 7-9 (30-day) layers of vascular cambial cells that could be identified histologically (Extended Data Fig. 1b). In each radial cell file, three different types of GUS sectors were observed (Fig. 1c, Extended Data Fig. 5 a-c). GUS clones that originated from the xylem (green) or phloem (yellow) side of the cambium differentiated into the corresponding cell types or divided before differentiation. In contrast, cells located in the centre of the cambium (red) produced broad GUS sectors spanning into the differentiated xylem and phloem (Fig. 1 c, d, Extended Data Fig. 5 c, d). Similarly, induction of clones in the ANT expression domain, marking a narrow layer of dividing cells, led to production of GUS sectors spanning to both directions (Fig. 2a, Extended Data Fig. 5e-h). These data demonstrate that the vascular cambium consists of a single, bifacial stem cell in each radial cell file, marked with ANT expression. These stem cells produce both xylem and phloem, thus confirming the 145 -year-old theory of uniseriate vascular cambium ${ }^{3,4}$, at least in the Arabidopsis root. This theory states that a single layer of initials (stem cells) produces both xylem and phloem; and was later elaborated by the observation that the position of the 'initials' varied between neighbouring radial files $^{1,4}$. Our experimental data support these later modifications; the variable location of the stem cells can be observed as a considerable radial distribution of the GUS sectors originated from stem cells (Fig. 1d, Extended Data Fig. 5d).

To test whether the HD-ZIP IIIs are sufficient to induce the formation of an ectopic organiser within the cambium, we inducibly overexpressed an miR165/166-resistant version of AtHB8 (AtHBd) in the stem cell domain under the ANT promoter $^{12}$. Induction of $p A N T: X V E>>A t H B 8 d-Y F P$ rapidly inhibited cell division in the stem cells, thus confirming the ability of the HD-ZIP IIIs to promote cellular quiescence (Extended Data Fig. 6a-c). This local quiescence was associated with a centrifugal shift (i.e. towards phloem) in the domain of high cell division and organiser marker expression (Extended Data Fig. 6b, d), followed by the formation of ectopic xylem vessels in the cambium (Extended Data Fig. 6e). These data demonstrate that at least AtHB8 is sufficient to induce organiser function within the vascular cambium.

Auxin promotes expression of HD-ZIP IIIs in vascular tissues during primary development, and their expression often correlates with auxin signalling maxima ${ }^{18,19}$. Since the auxin maximum peaks on the xylem-side of tree vascular cambia ${ }^{20,21}$, we next studied the auxin signalling response pattern in the Arabidopsis root cambium. Auxin response markers showed a local maximum in the xylem domain, tapering off towards the cambium stem cells, thus resembling the expression pattern of AtHB8, WOX4 and PXY (Figs 2a, 3a; Extended Data Fig. 7a, b). Auxin signalling is mediated by AUXIN RESPONSE FACTOR (ARF) transcription factors ${ }^{22}$. We focused our expression analysis on class A ARFs, since their activity is preferentially auxin regulated ${ }^{22}$, and the 
class A ARF ARF5/MONOPTEROS (MP) has been shown to regulate proper vascular formation ${ }^{23}$ and cambium cell division by controlling the expression of $W O X 4^{24}$. Of the five class A ARFs, three (ARF5/MP, ARF7 and ARF19) showed strong and consistent expression in the vascular cambium (Extended Data Fig. 7c, d). Since the $\operatorname{arf7,19}$ double mutant did not show clear patterning defects (Extended Data Fig. 8a), and strong $m p$ mutants failed to initiate roots $^{25}$, we developed an inducible artificial microRNA line targeting MP (amiMP) in the arf7,19 background. Ubiquitous induction of amiMP led to a reduction in MP transcript levels, downregulation of WOX4 and a subset of HD-ZIP IIIs, and a reduction in secondary vessel formation (Fig. 3b, c; Extended Data Fig. 8b, c), similar to the miR165a overexpression phenotype (Fig. 2b). Similarly, ubiquitous induction of an inducible, stable ARF-signalling repressor axr3-1 ${ }^{26}$ led to a strong downregulation of the organiser, stem cell and phloem identity genes, as well as the HD-Zip III genes, followed by termination of vascular cambium activity (Fig. 3d; Extended Data Fig. 8d-f). These data show that auxin signalling through MP, ARF7 and ARF19 and perhaps other ARFs promotes the expression of HD-ZIP IIIs, PXY and WOX4 in the stem cell organiser and that this signalling module is required to maintain vascular cambium identity. Since manipulations of HD-ZIP III levels lead to changes in auxin response (Extended data Fig. 7e-g), our data further suggest that auxin, ARFs and HD-ZIP IIIs are under a positive feedback regulation in the vascular cambium.

Finally, we tested whether the components determining the stem cell organiser are sufficient to induce vascular cambium formation when ectopically expressed. We generated activation clones in the phloem pole pericycle and surrounding cell files using an inducible S17 promoter ${ }^{12,16}$ (Extended Data Fig. 9a, b). Even though AtHB8d was sufficient to re-specify the organiser when overexpressed in stem cells of the vascular cambium, it was unable to induce xylem vessel formation or expression of cambial markers in the S17 domain (Extended Data Figs 6 and 9c, d). However, a proportion of clones expressing MPA, a dominant active version of $M P^{27}$, formed ectopic vessel elements and had periclinal cell divisions in the adjacent cells (Fig. 4a, Extended Data Fig. 9e, f). Time-course experiments revealed that MPA cell-autonomously induced WOX4, PXY and AtHB8 expression, followed by differentiation of the clones into vessel elements (Fig. 4a-c). During differentiation into vessels, MPA clones induced ectopic expression of WOX4, PXY and AtHB8 in the adjacent cells, indicating that MP can promote organiser cell identity also in a cell non-autonomous manner (Fig. 4b). Additionally, early phloem marker PEAR1 appeared ectopically at a distance of a few cells from the clone, suggesting that the presence of an MPA clone is sufficient to promote the formation of a vascular cambium (Fig. 4c). MPA induces expression of the genes that define the stem cell organiser before xylem vessel formation, suggesting that the cellular identity induced by high auxin signalling rather than the presence of xylem vessels defines the organiser. Supporting this, clonal activation of VASCULAR-RELATED NAC-DOMAIN 6 (VND6), which directly induces terminal differentiation of xylem vessels ${ }^{28}$, failed to promote organiser gene expression within the clone (Extended Data Figs 1a and 9g). In 
156

157

158

159

160

161

162

163

164

165

166

167

168

169

170

171

172

173

174

175

176

177

178

179

180

181

182

183

the longer term, a proportion of VND6-induced vessels were surrounded by cells expressing a subset of cambial markers; however, these cells rarely divided (Extended Data Fig. 9e, f). This indicates that the chemical or physical properties of terminally differentiated xylem vessels are not sufficient to induce organiser formation. Together, these data demonstrate that local high auxin signalling within root vascular tissue is sufficient to establish an organiser cell that promotes stem cell-like divisions and identity in the adjacent cells (Fig. 4d). Our data revealed a dynamic nature of the organiser; differentiation of the organiser into a xylem vessel leads to formation of a new organiser in the adjacent cambial stem cell, thus ensuring the maintenance of the vascular cambium (Extended Data Fig. 10).

We uncovered a mechanistic framework defining the stem cell organiser of the vascular cambium (Fig. 4d). Similarly to the cells in the stem cell organiser (quiescent centre) of the root apical meristem ${ }^{29}$, the cells in the cambium organiser are quiescent and are defined by an auxin response maximum. However, unlike the root quiescent centre, the stem cell organiser of the vascular cambium is dynamic, since as the root grows thicker, organiser cells differentiate and stem cells acquire organiser identity (Extended Data Fig. 10a). Cells of the organising centre of the shoot apical meristem also are replaced as they differentiate, although in that case the location of the organiser is stable. All three organisers express a WOX family member: WUSCHEL in the shoot apical meristem, WOX5 in the root meristem ${ }^{2}$ and WOX4 in the vascular cambium, underscoring the related identity of cells in the three locations. Future challenges are to identify the cell non-autonomous stem cell factors originating from the organiser cells (Fig. 4d), and to link other known cambial regulators ${ }^{30}$ to the signalling module defining the organiser (Extended Data Fig. 10).

\section{SUPPLEMENTARY INFORMATION}

Supplementary Tables 1 to 8

\section{ACKNOWLEDGEMENTS}

We thank T. Berleth, A. Carlsbecker, T. Greb, P. Ramachandran, E. Scarpella, D. Weijers for sharing published material; A.-M. Bågman, M. Herpola and J. Smetana for technical assistance; W. Yang for helping with the in situ protocol; S. el-Showk, P. Etchells, Y. Helariutta, S. Otero and B. Scheres for constructive comments. This work was supported by the Academy of Finland (grants \#132376, \#266431, \#271832), University of Helsinki HiLIFE 
184

185

186

187

188

189

190

191

192

193

194

195

196

197

198

199

200

201

202

203

204

205

206

207

208

209

210

fellowship (O.S., R.M., M.L., A.S.G., F.S.R., M.L.G., R.S., T.B., S.B., A.P.M.), Erasmus+ (F.S.R., M.L.G.), and U.S. National Science Foundation grant IOS-0744874 (M.-F.W, J.W.R.).

\section{AUTHOR CONTRIBUTIONS}

O.S. and A.P.M. designed the experiments. O.S. carried out all the experiments, except, R.M. and M.L. conducted qRT-PCR, amiRNA and inducible axr3 work; F.S.R analysed HD-ZIP III mutants and S.B. carried out RNA in situ hybridisation and lineage tracing with BAP. M.-F.W. and J.W.R generated and tested amiRNA plasmid. A.S.G. and M.L.G. provided data on the inducible miR165a line. A.A. conducted the statistical analyses. R.S., S.M., P.R. and T.B. provided transgenic lines for the study. O.S. and A.P.M. wrote the manuscript with the help of co-authors.

\section{COMPETING INTERESTS}

The authors declare no competing financial interests.

\section{CORRESPONDING AUTHOR}

Correspondence to Ari Pekka Mähönen: AriPekka.Mahonen@helsinki.fi

\section{REFERENCES}

1. Larson, P. R. The vascular cambium: development and structure. Berlin, Germany: Springer-Verlag. (1994).

2. Greb, T. \& Lohmann, J. U. Plant Stem Cells. Curr. Biol. 26, R816-21 (2016).

3. Sanio, C. Anatomie der gemeinen Kiefer (Pinus silvestris L.) II. Entwickelungsgeschichte der Holzzellen. Jahrb Wiss Bot 9, 50-128 (1873).

4. Evert, R. F. \& Eichhorn, S. E. Esau's plant anatomy: Meristems, cells, and tissues of the plant body: Their structure, function, and development: Third edition. , 1-601 (2006).

5. Chaffey, N., Cholewa, E., Regan, S. \& Sundberg, B. Secondary xylem development in Arabidopsis: a model for wood formation. Physiol. Plant. 114, 594-600 (2002).

6. Wunderling, A. et al. A molecular framework to study periderm formation in Arabidopsis. New Phytol. (2018). 
7. Atta, R. et al. Pluripotency of Arabidopsis xylem pericycle underlies shoot regeneration from root and hypocotyl explants grown in vitro. Plant J. 57, 626-644 (2009).

8. Carlsbecker, A. et al. Cell signalling by microRNA165/6 directs gene dose-dependent root cell fate. Nature 465, 316-321 (2010).

9. Ohashi-Ito, K. \& Fukuda, H. HD-zip III homeobox genes that include a novel member, ZeHB-13 (Zinnia)/ATHB15 (Arabidopsis), are involved in procambium and xylem cell differentiation. Plant Cell Physiol 44, 1350-1358 (2003).

10. Baima, S. et al. The arabidopsis ATHB-8 HD-zip protein acts as a differentiation-promoting transcription factor of the vascular meristems. Plant Physiol. 126, 643-655 (2001).

11. Robischon, M., Du, J., Miura, E. \& Groover, A. The Populus class III HD ZIP, popREVOLUTA, influences cambium initiation and patterning of woody stems. Plant Physiol. 155, 1214-1225 (2011).

12. Siligato, R. et al. MultiSite Gateway-Compatible Cell Type-Specific Gene-Inducible System for Plants. Plant Physiol. 170, 627-641 (2016).

13. Mallory, A. C. et al. MicroRNA control of PHABULOSA in leaf development: importance of pairing to the microRNA 5' region. EMBO J. 23, 3356-3364 (2004).

14. Hirakawa, Y., Kondo, Y. \& Fukuda, H. TDIF peptide signaling regulates vascular stem cell proliferation via the WOX4 homeobox gene in Arabidopsis. Plant Cell 22, 2618-2629 (2010).

15. Fisher, K. \& Turner, S. PXY, a receptor-like kinase essential for maintaining polarity during plant vasculartissue development. Curr. Biol. 17, 1061-1066 (2007).

16. Lee, J. Y. et al. Transcriptional and posttranscriptional regulation of transcription factor expression in Arabidopsis roots. Proc. Natl. Acad. Sci. U. S. A. 103, 6055-6060 (2006).

17. Miyashima, S. et al. Mobile PEAR transcription factors integrate positional cues to prime cambial growth. Nature (in press).

18. Ursache, R. et al. Tryptophan-dependent auxin biosynthesis is required for HD-ZIP III-mediated xylem patterning. Development 141, 1250-1259 (2014).

19. Donner, T. J., Sherr, I. \& Scarpella, E. Regulation of preprocambial cell state acquisition by auxin signaling in Arabidopsis leaves. Development 136, 3235-3246 (2009).

20. Uggla, C., Moritz, T., Sandberg, G. \& Sundberg, B. Auxin as a positional signal in pattern formation in plants. Proc. Natl. Acad. Sci. U. S. A. 93, 9282-9286 (1996).

21. Immanen, J. et al. Cytokinin and Auxin Display Distinct but Interconnected Distribution and Signaling Profiles to Stimulate Cambial Activity. Curr. Biol. 26, 1990-1997 (2016). 
22. Weijers, D. \& Wagner, D. Transcriptional Responses to the Auxin Hormone. Annu. Rev. Plant. Biol. 67, 539574 (2016).

23. Przemeck, G. K., Mattsson, J., Hardtke, C. S., Sung, Z. R. \& Berleth, T. Studies on the role of the Arabidopsis gene MONOPTEROS in vascular development and plant cell axialization. Planta 200, 229-237 (1996).

24. Brackmann, K. et al. Spatial specificity of auxin responses coordinates wood formation. Nat. Commun. 9 , 875-018-03256-2 (2018).

25. Berleth, T. \& Jurgens, G. The role of the monopteros gene in organising the basal body region of the Arabidopsis embryo. Development 118, 575-587 (1993).

26. Mahonen, A. P. et al. PLETHORA gradient formation mechanism separates auxin responses. Nature 515, 125-129 (2014).

27. Krogan, N. T., Ckurshumova, W., Marcos, D., Caragea, A. E. \& Berleth, T. Deletion of MP/ARF5 domains III and IV reveals a requirement for Aux/IAA regulation in Arabidopsis leaf vascular patterning. New Phytol. 194, 391-401 (2012).

28. Kubo, M. et al. Transcription switches for protoxylem and metaxylem vessel formation. Genes Dev. 19, 1855-1860 (2005).

29. Sabatini, S. et al. An auxin-dependent distal organizer of pattern and polarity in the Arabidopsis root. Cell 99, 463-472 (1999).

30. Nieminen, K., Blomster, T., Helariutta, Y. \& Mahonen, A. P. Vascular Cambium Development. Arabidopsis Book 13, e0177 (2015).

\section{METHOD AND EXTENDED DATA FIGURE REFERENCES}

31. Emery, J. F. et al. Radial patterning of Arabidopsis shoots by class III HD-ZIP and KANADI genes. Curr. Biol. 13, 1768-1774 (2003).

32. Heidstra, R., Welch, D. \& Scheres, B. Mosaic analyses using marked activation and deletion clones dissect Arabidopsis SCARECROW action in asymmetric cell division. Genes Dev. 18, 1964-1969 (2004).

33. Wu, M. F., Tian, Q. \& Reed, J. W. Arabidopsis microRNA167 controls patterns of ARF6 and ARF8 expression, and regulates both female and male reproduction. Development 133, 4211-4218 (2006).

34. Karimi, M., Depicker, A. \& Hilson, P. Recombinational cloning with plant gateway vectors. Plant Physiol. 145, 1144-1154 (2007). 
272

273

274

275

276

277

278

279

280

281

282

283

284

285

286

287

288

289

290

291

292

293

294

295

296

297

298

299

300

301

302

303

35. Prigge, M. J. et al. Class III homeodomain-leucine zipper gene family members have overlapping, antagonistic, and distinct roles in Arabidopsis development. Plant Cell 17, $61-76$ (2005).

36. Okushima, Y. et al. Functional genomic analysis of the AUXIN RESPONSE FACTOR gene family members in Arabidopsis thaliana: unique and overlapping functions of ARF7 and ARF19. 17, 444-463 (2005).

37. Sawchuk, M. G., Edgar, A. \& Scarpella, E. Patterning of leaf vein networks by convergent auxin transport pathways. PLoS Genet. 9, e1003294 (2013).

38. Friml, J. et al. Efflux-dependent auxin gradients establish the apical-basal axis of Arabidopsis. Nature 426, 147-153 (2003).

39. Liao, C. Y. et al. Reporters for sensitive and quantitative measurement of auxin response. Nat. Methods 12, 207-10, 2 p following 210 (2015).

40. Baesso, B. et al. Transcription factors PRE3 and WOX11 are involved in the formation of new lateral roots from secondary growth taproot in A. thaliana. 20, 426-432 (2018).

41. Suer, S., Agusti, J., Sanchez, P., Schwarz, M. \& Greb, T. WOX4 imparts auxin responsiveness to cambium cells in Arabidopsis. Plant Cell 23, 3247-3259 (2011).

42. Vidaurre, D. P., Ploense, S., Krogan, N. T. \& Berleth, T. AMP1 and MP antagonistically regulate embryo and meristem development in Arabidopsis. Development 134, 2561-2567 (2007).

43. Wilmoth, J. C. et al. NPH4/ARF7 and ARF19 promote leaf expansion and auxin-induced lateral root formation. Plant J. 43, 118-130 (2005).

44. Bonke, M., Thitamadee, S., Mahonen, A. P., Hauser, M. T. \& Helariutta, Y. APL regulates vascular tissue identity in Arabidopsis. Nature 426, 181-186 (2003).

45. Zhong, R., Lee, C., Zhou, J., McCarthy, R. L. \& Ye, Z. H. A battery of transcription factors involved in the regulation of secondary cell wall biosynthesis in Arabidopsis. Plant Cell 20, 2763-2782 (2008).

46. Baima, S. et al. The expression of the Athb-8 homeobox gene is restricted to provascular cells in Arabidopsis thaliana. Development 121, 4171-4182 (1995).

47. Idanheimo, N. et al. The Arabidopsis thaliana cysteine-rich receptor-like kinases CRK6 and CRK7 protect against apoplastic oxidative stress. Biochem. Biophys. Res. Commun. 445, 457-462 (2014).

48. Kurihara, D., Mizuta, Y., Sato, Y. \& Higashiyama, T. ClearSee: a rapid optical clearing reagent for whole-plant fluorescence imaging. Development 142, 4168-4179 (2015).

49. Kotogany, E., Dudits, D., Horvath, G. V. \& Ayaydin, F. A rapid and robust assay for detection of S-phase cell cycle progression in plant cells and tissues by using ethynyl deoxyuridine. Plant. Methods 6, 5-4811-6-5 (2010).

50. Randall, R. S. et al. AINTEGUMENTA and the D-type cyclin CYCD3;1 regulate root secondary growth and respond to cytokinins. Biol. Open 4, 1229-1236 (2015). 
51. Liebsch, D. et al. Class I KNOX transcription factors promote differentiation of cambial derivatives into xylem fibers in the Arabidopsis hypocotyl. Development 141, 4311-4319 (2014).

52. Czechowski, T., Stitt, M., Altmann, T., Udvardi, M. K. \& Scheible, W. R. Genome-wide identification and testing of superior reference genes for transcript normalization in Arabidopsis. Plant Physiol. 139, 5-17 (2005).

53. Vandesompele, J. et al. Accurate normalization of real-time quantitative RT-PCR data by geometric averaging of multiple internal control genes. Genome Biol. 3, RESEARCH0034 (2002).

54. Livak, K. J. \& Schmittgen, T. D. Analysis of relative gene expression data using real-time quantitative PCR and the 2(-Delta Delta C(T)) Method. Methods 25, 402-408 (2001).

55. Yang, W., Wightman, R. \& Meyerowitz, E. M. Cell Cycle Control by Nuclear Sequestration of CDC20 and CDH1 mRNA in Plant Stem Cells. Mol. Cell 68, 1108-1119.e3 (2017).

56. Mahonen, A. P. et al. Cytokinin signaling and its inhibitor AHP6 regulate cell fate during vascular development. Science 311, 94-98 (2006).

57. Colon-Carmona, A., You, R., Haimovitch-Gal, T. \& Doerner, P. Technical advance: spatio-temporal analysis of mitotic activity with a labile cyclin-GUS fusion protein. Plant J. 20, 503-508 (1999).

58. Bishopp, A. et al. A mutually inhibitory interaction between auxin and cytokinin specifies vascular pattern in roots. Curr. Biol. 21, 917-926 (2011).

59. De Rybel, B. et al. Plant development. Integration of growth and patterning during vascular tissue formation in Arabidopsis. Science 345, 1255215 (2014).

60. Ohashi-Ito, K. et al. A bHLH complex activates vascular cell division via cytokinin action in root apical meristem. Curr. Biol. 24, 2053-2058 (2014).

61. Matsumoto-Kitano, M. et al. Cytokinins are central regulators of cambial activity. Proc. Natl. Acad. Sci. U. S. A. 105, 20027-20031 (2008).

\section{FIGURE LEGENDS}

Figure 1 | Lineage tracing revealed the origin of the vascular cambium stem cells. a, The activation of secondary root growth is characterised by the initiation of periclinal cell divisions in procambial cells (black arrowheads), followed by divisions in pericycle (white arrowheads), leading to the formation of vascular cambium and phellogen. Cell divisions are marked only in upper half of the cross section. $\mathbf{b}$, Lineage tracing initiated from the primary vasculature (top) showed the importance of an initial xylem connection for the ontogeny of the cambium (yellow dashed line). The bottom panel shows the four major types of clonal sectors obtained. Frames are coloured to match with the corresponding cell lineages in the top panels. The numbers in the fate map (bottom 
right) refer to the observed number of the sectors. $\mathbf{c}, \mathbf{d}$, Lineage tracing in active cambium revealed a common stem cell for xylem and phloem. GUS sectors (black bars) plotted based on the position of thinnest cell wall (arrow) in each radial file, representing the most recent periclinal cell division in cambium (y value $=0$ ). Scale bars represent $50 \mu \mathrm{m}$. All experiments were repeated four times.

Figure 2 | HD-ZIP Ills determine the organiser of the vascular cambium. a, Confocal microscopy of cross sections of 14-day old ANT double markers. White arrows indicate pairs of nuclei in recently dividing cells. Insets, merged reporter channels. b, Bright field microscopy of root after miR165a induction (Ind) for 6 days (in 5-day old plants). Red dots, secondary xylem vessels (SXV); quantification presented on the right. Box-and-whisker plot shows median and interquartile range; whiskers show range, dots outliers. Two-tailed Wilcoxon test. $n$, independent roots. c, Confocal images of EdU staining reveal a centripetal shift of the cell division domain after 2-day miR165a induction (in 8-day old plants). Asterisks mark cambium. Histograms showing the relative distances (RD) of EdUpositive nuclei in the radial direction from the root midpoint (xylem axis: $x$ value $=0$, periderm: $x$ value $=1$ ), as depicted in Mock. $n_{n}$, total number of nuclei. d, Confocal microscopy of fluorescent markers after 2-day miR165a induction (in 8-day old plants). Dashed lines, borders of the secondary tissues. The numbers in the bottom left corner of subpanels represent the frequency of the observed phenotype. a, c, d, Cell wall staining in grey. p, phloem; $x$ xylem. All experiments were repeated at least three times. Scale bars represent $50 \mu \mathrm{m}$.

Figure 3 | Auxin signalling is required for xylem differentiation and organizer identity. a, Confocal microscopy of DR5:nYFP and CAtHB8-YFP (heatmap) root cross sections. Nuclear fluorescence signal (circles indicate examples of ROIs) was quantified in three cell layers from the most recent cell division (arrowheads) towards xylem (positions $-1,-2,-3$ ) and phloem (positions 1, 2, 3). $n_{n}=$ total number of nuclei. Cell wall staining in grey. p, phloem; $x$, xylem. b, qRT-PCR analysis of marker gene expression in amiMP T1 transformants with the lowest MP transcript levels. Each point represents an individual amiMP T1 transformant $(n=10)$ or individual $\operatorname{arf7,19}$ plant $(n=5)$ of the same age. The $p$-values of a one-sided t-test comparing amiMP to arf7,19 controls are provided under each gene name, and significant values are in bold-face. c, Bright field microscopy of pUBQ10:XVE >>amiMP transformants in the arf7,19 background induced for 7 days (in 5-day old plants). Yellow dots, secondary xylem vessels. Experiment was repeated three times with similar results. $\mathbf{d}$, qRT-PCR analysis of marker gene expression in inducible axr3-1 after 24h induction (in 8-day old plants). Data are from three experiments ( $n=16$ roots per treatment). In a and $\mathbf{d}$, box-and-whisker plots show median and interquartile range; 
365

366

367

368

369

370

371

372

373

374

375

376

377

378

379

380

381

382

383

384

385

386

387

388

389

390

391

392

393

whiskers show range. One-tailed t-test. In a, dots represents independent data points. Scale bars represent 50 $\mu \mathrm{m}$.

Figure 4 | MP is sufficient for the formation of an ectopic organiser. a, Bright field micrograph of an MPA-GUS clone with periclinal cell divisions induced cell non-autonomously. Red dots, GUS-negative cells dividing periclinally around the MPA-GUS clone. $\mathbf{b}$, Confocal analysis of marker expression within MPA-YFP clones (top) and around MPA-YFP-induced ectopic vessels (bottom). Cell wall staining in grey. Insets show YFP channel except for insets of PXY and AtHB8 in bottom panels showing RFP channel. c, PEAR1 is induced a few cell layers from MPA-induced ectopic vessels (asterisk). An arrow indicates a gap between ectopic vessel and PEAR1 expression. d, A model illustrating how a local auxin maximum and the downstream components define an organiser which positions the stem cell in the adjacent cell. Curved arrows represent bidirectional cell proliferation from the bifacial stem cell. The numbers in the left corner of subpanels represent the frequency of the observed phenotype. Scale bars represent $50 \mu \mathrm{m}$ unless indicated otherwise.

\section{METHODS}

\section{Gene accession numbers}

CYCB1;1, AT4G37490; VND6, AT5G62380; WOX4, AT1G46480; PXY/TDR, AT5G61480; EPM/PEAR1/S29/Dof2.4, AT2G37590; ANT, AT4G37750; AtHB8, AT4G32880; CNA/AtHB15, AT1G52150; PHB, AT2G34710; PHV, AT1G30490; REV, AT5G60690; mir165a, AT1G01183; MP/ARF5, AT1G19850; ARF6, AT1G30330; ARF7, AT5G20730; ARF8, AT5G37020; ARF19, AT1G19220; APL, AT1G79430; S17, AT2G22850.

\section{Plant material and cloning}

Most of the entry vectors were generated by PCR amplification of the desired DNA sequence with the primers indicated in Table S1 and recombination of the purified PCR product into MultiSite Gateway compatible pDONR entry vectors (Table S2). Obtained PCR products were amplified in two steps (marked with asterisks in Table S2), first with gene-specific primers with partial attB overhangs and then with adaptor primers to generate PCR products with full length attB recombination sequences. The dominant version of cAtHB8 (AtHB8d) has a mutation (CCG $\rightarrow$ CTG) in the last codon of the miR165a-recognition sequence, similar to the dominant REVOLUTA mutant rev-10d ${ }^{31}$. The entry vector p221z-c AtHB8d(-)stop was generated by site-directed mutagenesis using 
394

395

396

397

398

399

400

401

402

403

404

405

406

407

408

409

410

411

412

413

414

415

416

417

418

419

420

421

422

423

p221z-c AtHB8(-)stop as a template and primers AtHB8dF1 and AtHB8dR1 using the QuikChange II Site-Directed Mutagenesis Kit (Agilent Technologies). The entry vector p221a-CYCB1;1-CRE was made by PCR amplification of CRE recombinase ${ }^{32}$ from p221z-CRE1 using the CreMfeF and CreMfeR primers, and the resulting PCR product was ligated into the unique Mfel restriction site of p221a-CycB1-dbox ${ }^{26}$. MicroRNA against MONOPTEROS (amiMP) was obtained by PCR mutagenising the MIR167a precursor sequences (At3g22886) ${ }^{33}$ using the following primers: aMIR-MP_1, aMIR-MP_2, aMIR-MP_3 and aMIR-MP_4 (sequences in Table S3). amiMP was then cloned into pENTR/D_TOPO to create pENT-AtMIR167a-MP. To construct gANT-GFP, genomic fragment of ANT was amplified by using primers ANT(-)5172 and ANT(+)2885R, and cloned into pAN19 (a modified pUC18). GFP was inserted into the C-terminus of $A N T$ coding sequence by using inverse PCR. The resultant $g A N T$-GFP fragment was re-cloned into pBIN30 (a modified pBIN19) binary vector. pSm43GW was generated from pHm43GW in two steps. First, hygromycin resistance was replaced with a -SphI-YFP-Kpn1- PCR fragment using the YFPfrg_F1 and YFPfrg_R2 primers and the Sphl and $K p n l$ restriction sites to obtain pYm43GW. Next, the YFP fragment was replaced with the sulfadiazine resistance gene amplified from GABI_874H08 line gDNA with primers sulf-KpnIF and sulf-SnaBIR, and the resulting PCR product was cloned into pYm43GW using the Kpnl and Stul restriction sites.

MultiSite Gateway technology (Invitrogen) was used to combine promoter ( $1^{\text {st }}$ box), gene of interest $\left(2^{\text {nd }}\right.$ box) and tag or terminator ( $3^{\text {rd }}$ box) Gateway entry clones with Gateway-compatible binary destination vectors ${ }^{12,34}$ in a MultiSite LR Clonase reaction. All expression vectors generated in this study are listed in Table S4.

All constructs were dipped into the Col-0 ecotype, and single insertion lines were screened based on Mendelian segregation of a selection marker. Several lines for each newly made construct were used for phenotype observation. Plant material produced in this study as well as published lines provided by others are indicated in Table S5. The following transgenic and mutant lines have been described elsewhere: cna-2 phb-13 phv-11; athb811 cna-2 phb-13 phv-11 ${ }^{8,35}$; arf7-1 arf19-1 ${ }^{36}$; DR5rev::YFPnuc ${ }^{37}$, DR5rev:erGFP ${ }^{38}$, DR5V2:n3GFP ${ }^{39}$, DR5rev:GUS ${ }^{40}$, pWOX4:erYFP ${ }^{41}$, pMP:MP-GUS ${ }^{42}$, pARF7:GUS \& pARF19:GUS ${ }^{43}$, gARF8-GUS \& gARF6-GUS ${ }^{33}, p G 1090: X V E>>a x r 3-$ 1-RFP ${ }^{26}, p A P L: G U S^{44}, g V N D 6-G U S^{45}$ and $p A t H B 8: G U S^{46}$.

\section{Plant growth and chemical treatments}

For almost all experiments, seeds were surface-sterilised in $70 \%$ ethanol, then in $20 \%$ chlorine, washed twice in $\mathrm{H}_{2} \mathrm{O}$, and plated on half strength $\mathrm{GM}$ medium ( $\left.1 / 2 \mathrm{GM}\right)$ containing $0.5 \times$ MS salt mixture with vitamins (Duchefa), $1 \%$ sucrose, $0,5 \mathrm{~g} / \mathrm{I} \mathrm{MES} \mathrm{pH} 5.8$ and $0.8 \%$ agar and vernalised in $4^{\circ} \mathrm{C}$ for 2 days. The age of the seedlings was measured from the moment they were placed in a vertical position in the growth cabinet, where they were 
424

425

426

427

428

429

430

431

432

grown at $22^{\circ} \mathrm{C}$ under long day conditions. For experiment with soil-grown plants, seeds were directly placed on soil and grown under the same conditions.

17- $\beta$-oestradiol (Sigma), a synthetic derivative of oestrogen, and 6-Benzylaminopurine (BAP; Sigma), a cytokinin, were prepared as $20 \mathrm{mM}$ stock solutions in DMSO and stored at $-20^{\circ} \mathrm{C}$. 5-Ethynyl-2' -deoxyuridine, a thymidine analogue (EdU; Thermo Fisher), was prepared as a $10 \mathrm{mM}$ stock solution in DMSO and stored at $4^{\circ} \mathrm{C}$. XVE-based gene induction ('Ind' in figure panels) was carried out by transferring plants to plates supplemented with $5 \mu \mathrm{M}$ $17-\beta$-oestradiol or an equal volume of DMSO ('Mock' in figure panels) unless otherwise indicated. The BAP working concentration was $0.1 \mu \mathrm{M}$. For EdU treatment, plants were transferred into $1 / 2$ GM liquid medium supplemented with $10 \mu \mathrm{M}$ EdU and, eventually, with inducer or mock for $16 \mathrm{~h}$. Unless stated otherwise, the age of the seedlings at the start of the treatment is indicated in the brackets in the figure legends.

\section{GUS staining, microtome sectioning and histology}

GUS detection and histology analysis were performed according to ${ }^{47}$ with slight modifications. First, samples were vacuum-infiltrated with the GUS staining solution for $1 \mathrm{~h}$ and then incubated at $37^{\circ} \mathrm{C}$ until the desired level of GUS staining was obtained ( $2 \mathrm{~h}$ - overnight). Samples were cut on a microtome in $10 \mu \mathrm{m}$ sections from $5 \mathrm{~mm}$ below the root-hypocotyl junction. For histological analysis of clones, the upper 1-1.5 cm root segment was cut every $200 \mu \mathrm{m}$ to avoid repeated analysis of the same cells. Finally, root sections were stained for $5 \mathrm{~s}$ in a $0.05 \%$ (w/v) Ruthenium Red solution (Fluka Biochemika) or a $0.05 \%$ (w/v) Toluidine blue solution in deionised water. To stain phloem companion cells, a $0.5 \%$ (w/v) Safranin solution in $50 \%$ ethanol and a $1 \%$ (w/v) Alcian blue aqueous solution were used. All histological stainings used in each figure are indicated in Table S6.

\section{Fluorescent marker analysis, vibratome sectioning and EdU detection}

To analyse fluorescent reporters from a lateral view, roots were fixed in 4\% paraformaldehyde (PFA, Sigma) in 1 x PBS pH 7.2 overnight, washed twice in 1 x PBS and cleared in ClearSee solution ${ }^{48}$ supplemented with $1 \mu \mathrm{g} / \mathrm{ml}$ Calcofluor white (Sigma) for at least three days.

Vibratome sectioning was used for fluorescent marker analysis and EdU detection. Roots were fixed in 4\% PFA in $1 \times$ PBS pH 7.2 for $1 \mathrm{~h}$ under vacuum, washed in ice-cold $1 \times$ PBS and embedded in $4 \%$ agarose in $1 \times$ PBS. Samples were cut on a vibratome in $200 \mu \mathrm{m}$ sections from $5 \mathrm{~mm}$ below the root-hypocotyl junction. Obtained agarose slices containing root cross sections were washed twice in 1 x PBS, cell walls were stained with $1 \mu \mathrm{g} / \mathrm{ml}$ Calcofluor white (Sigma) in 1 x PBS for $3 \mathrm{~h}$ - overnight and finally cleared with ClearSee solution. To visualise EdU 
453

454

455

456

457

458

459

460

461

462

463

464

465

466

467

468

469

470

471

472

473

474

475

476

477

478

479

480

481

positive nuclei with the Click-iT ${ }^{\mathrm{TM}}$ EdU Alexa Fluor ${ }^{\mathrm{TM}} 488$ Imaging Kit (Thermo Fisher), fixed cross sections were washed twice in $1 \times$ PBS and then incubated in a modified EdU detection cocktail ${ }^{49}$ at RT for $1 \mathrm{~h}$, washed twice in $1 \times$ PBS and stained with $1 \mu \mathrm{g} / \mathrm{ml}$ Calcofluor or DAPI solution in 1 x PBS.

\section{Microscopy and image processing}

Histological cross sections were imaged using a Leica 2500 microscope (20x and 40x objectives). Confocal analysis was carried out with Leica SP5 and SP8 microscopes. Confocal images were acquired with the Leica LAS AF Software using ClearSee, 1x PBS or water as an imaging medium. All combinatorial fluorescence analyses were run in sequential scan mode. Confocal settings may have varied between experiments but always remained the same for an experimental sample and its corresponding control. However, to better visualise calcofluor-stained cell walls, the signal was sometimes unevenly adjusted between the sample and the control. EdU images represent maximal projections of five consecutive optical sections captured each $5 \mu \mathrm{m}$ in the z-direction.

Microsoft PowerPoint with image compression mode off was used to compile all the figures and to modify brightness and contrast, which was always identically applied to sample and control pictures. Images in Figure 1a were cropped and rotated using Corel PHOTO PAINT X6, which occasionally resulted in empty corners; for aesthetical reasons, these were filled with a triangle of a similar colour as the image background.

\section{Marker expression quantification, EdU and xylem vessel histograms}

Changes in marker expression were analysed from the lateral view; optical sections were captured in the middle of the root, which was determined by the root edges being in focus in the transmitted light channel. The data represent the relative width of the fluorescent domain compared with the root diameter. DR5:nYFP, DR5V2:nGFP and CAtHB8-YFP fluorescence were captured as one stack of 16-bit confocal images from 15 cross sections. The signal intensities of fluorescent nuclei were measured in ImageJ using Multi-point tool with mark width set to 15 pixels. Relative intensities were determined by comparing the signal with a brightest value in the whole dataset. For EdU and vessel distribution histograms, relative distances of EdU nuclei/vessels were determined by comparing their distance from the centre of xylem axis with the radius of the root. If not indicated otherwise, 25 cross sections were analysed. Histograms represent frequencies of EdU nuclei/vessels along the radial axes divided into 10 bins in percentage. Distance measurements were done using Leica Lite or ImageJ.

\section{amiMP analysis}

Due to persistent silencing of $p U B Q 10: X V E>>\operatorname{amiMP}(\operatorname{arf7-1,19-1)}$ in the $\mathrm{T} 2$ and $\mathrm{T} 3$ generations, individual plants were analysed in the T1 generation. Transformants in the arf7, 19 or wild-type backgrounds were grown on 
482

483

484

485

486

487

488

489

490

491

492

493

494

495

496

497

498

499

500

501

502

503

504

505

506

507

508

509

510

511

plates with $20 \mu \mathrm{g} / \mathrm{ml}$ phosphinothricin (basta, Duchefa) for 5 days without noticeable phenotypes caused by the selection, and then induced together with the controls for 7 days. Segments of the upper $2 \mathrm{~cm}$ of the root were collected for histological analysis, and the remaining (lower) part was used for RNA isolation. Since arf7-1,19-1 does not produce lateral roots, lower segment contained RNA exclusively from the primary root. Cell divisions were counted as the number of cells in one radial cell file between primary phloem pole and xylem axis.

\section{qRT-PCR}

RNA was extracted from the top $2 \mathrm{~cm}$ of the main root with lateral roots removed using the GeneJET Plant RNA purification kit (Thermo Fisher) and treated with DNase (Thermo Fisher). cDNA was synthetized from $100 \mathrm{ng}$ (AtHB8d and miR165a overexpressing plants), $500 \mathrm{ng}$ (axr3-1 overexpressing plants and miR165a-ox for PXY) or $50 \mathrm{ng}$ (amiMP plants) of RNA using MAXIMA H Minus reverse transcriptase (Thermo Fisher) with Oligo(dT) primers (Thermo Fisher). The PCR reaction was done on a Bio-Rad CFX384 cycler using EvaGreen qPCR mix (Solis Biodyne) and the following program: $10 \mathrm{~min}$ at $95^{\circ} \mathrm{C}, 50$ cycles $\left(10 \mathrm{~s}\right.$ at $95^{\circ} \mathrm{C}, 10 \mathrm{~s}$ at $60^{\circ} \mathrm{C}, 30 \mathrm{~s}$ in $\left.72{ }^{\circ} \mathrm{C}\right)$. All primers used in qRT-PCR are listed in Table S6: primers for $A C T 2, U B Q 10$ and $A N T^{50} ; P H B, P H V, A t H B 8$ and $C N A^{8}$;

$P X Y^{51}$; and TIP41 ${ }^{52}$ were described before. Results were normalized following the method of Vandesompele et al. $(2002)^{53}$ and Livak \& Schmittgen $(2001)^{54}$ to the reference genes ACT2, UBQ10 and TIP41. Three biological replicates were used for each line and time point, as well as three technical replicates, except in the analysis of amiMP lines (see above).

\section{RNA in situ hybridisation}

Primers used for RNA probe synthesis were published previously ${ }^{8}$. Sample preparation, sectioning, probe synthesis using T7 RNA polymerase (Thermo Fisher) and hybridization were performed as described ${ }^{55}$ with slight modifications. The uppermost $1 \mathrm{~cm}$ segment of the main root was cut from 21-day old soil-grown plants, and the tissues were vacuum infiltrated in FAA (50\% ethanol; $5 \%$ acetic acid; $3.7 \%$ formaldehyde) for 30 min and then fixed overnight at $4{ }^{\circ} \mathrm{C}$. For paraffin embedding, a Leica ASP200 vacuum tissue processor was used, following the protocol indicated in Table S7.

\section{Clone induction}

To induce clones before the onset of secondary growth, plates with 4-day old seedlings grown on $1 / 2 \mathrm{GM}$ plates with or without BAP were heat shocked at $37^{\circ} \mathrm{C}$ for 14 or $17 \mathrm{~min}$, immediately cooled down at $4^{\circ} \mathrm{C}$ for 15 min and further grown on new $1 / 2$ GM plates under normal growth conditions until GUS staining and histological analysis. For lineage tracing in active cambium, plants were grown for 15 days prior to heat shock. Clonal activation of pUAS:MPA-GUS (Fig. 4a) was carried out in the same way and the clones were analysed 6 days later. For lineage 
512 tracing in one-month old plants, whole $5 \mathrm{~cm}$ pots with individual plants where transferred into $37^{\circ} \mathrm{C}$ water bath 513 for $15 \mathrm{~min}$ followed by a cooling step under cold tap water for $1 \mathrm{~min}$. Clone activation in the phloem pole pericycle 514 and surrounding cells (S17 domain) was performed by transferring 5-day old seedlings into liquid $1 / 2$ GM medium 515 with 17- $\beta$-oestradiol for 8 or 16 min followed by four 15 min washes in medium without an inducer. After that, 516 plants were placed back on agar plates and grown under normal conditions. ANT:XVE>>CRE transformants in 517 35S:lox-GUS ${ }^{++}$background were selected on plates with hygromycin and claforan. After four days, hygromycin518 resistant plants were transferred on plates only with claforan and grown further without noticeable phenotype 519 caused by antibiotics. Clones in ANT domain were induced in a similar way as S17 clones with slight modifications: 520 17-day old plants were incubated with an inducer for 5 or $20 \mathrm{~min}$ and after washing they were placed on agar 521 plates with claforan. To increase the proportion of clones originating from single cells, roots were pre-screened 522 and only those with a low amount of clones were analysed further.

\section{Fate map and lineage tracing quantification}

524 Because the position of major cell types in the primary root vasculature is invariant, the fate map was generated 525 by counting the frequencies of different GUS sectors and grouping them into three categories: i) the most 526 frequent "basic sectors", ii) "large sectors" partially sharing clonal boundaries with two or more basic sector 527 types at once and iii) "half sectors" consisting a subdomain of one of the basic sector types. Only "basic sectors" 528 are presented in the fate map (Fig. 1b). BAP-mediated promotion of procambium formation in the place of 529 protoxylem is not a fully penetrant phenotype ${ }^{56}$, and therefore the presence [(+) protoxylem in Extended Data 530 Fig. 2e, f] or absence [(-) protoxylem] of protoxylem was observed before analysing the sectors.

531 Quantification of GUS sectors in active cambium was carried out by counting the cellular distance of sector 532 boundaries [xylem side $(y<0)$; phloem side $(y>0)$ ] from periclinally dividing cambial cells $(y=0)$ in the radial 533 direction. Dividing cambial cells were identified histologically based on the presence of a thin cell wall. In rare 534 cases where two dividing cambial cells were in given cell file, the distance was counted from the cell wall between 535 those cells. Only atypically large GUS sectors, sectors with unclear borders or located far from the vascular 536 cambium (Extended Data Fig. 5a, b, f) were excluded from quantification. Each bar in graphs (Fig. 1d, Extended

537 Data Fig. 5d, h) represents the size and position of an individual sector; bars with extensions denote sectors 538 beginning or ending with a xylem vessel.

\section{Quantification of MPA and VND6 clones}

540 The cell-autonomous effects of MPA and VND6 clones on cambial marker gene induction were measured as the 541 number of independent YFP-positive nuclei showing marker expression within the clones two days after 
542

543

544

545

546

547

548

549

550

551

552

553

554

555

556

557

558

559

560

561

562

563

564

565

566

567

568

569

570

induction. The cell non-autonomous effects were quantified as the number of ectopic vessels showing marker expression in the surrounding cells seven days after induction. At least 10 roots per genotype were quantified. The cell non-autonomous effect of MP on periclinal cell division was analysed by counting the frequency of periclinal cell divisions induced 16 days after germination in the GUS-negative cells surrounding the MPA-GUS clones/ectopic vessel. In these experiments (Fig. 4a; Extended Data Fig. 9e), periclinal cell divisions refer to cell division planes parallel to the surface of the neighbouring MPA-/VND6-induced vessels/clones. Only clearly ectopic vessels/clones (i.e. outside vascular cambium) were analysed further.

\section{General methodology and statistical analysis}

Unless otherwise stated, the number of individual plants, root sections or clones analysed is indicated as " $n$ " in Figure panels or legends. The fraction " $X / Y$ " in the corner of certain figure panels denotes the frequency of the presented phenotype. Statistical analyses were all carried out in R version 3.5.0 [http://www.r-project.org/]. For the mean comparison analysis, first the Shapiro-Wilk test for normality was performed and, upon failing to reject or rejecting this hypothesis, the appropriate one- or two-sided Student t-test or the nonparametric Wilcoxon rank sum test was performed, respectively. Regression analyses were performed following the affirmation of the normality assumption of the residuals. In general, we considered 0.05 as the significance threshold for $P$-values. The Kullback-Leibler divergent distance was used to quantify the level of dissimilarity between histograms, and, finally, the mean between the two directional values is reported. For all boxplots, the centre line represents the median and the lower and upper limits of boxes indicates the $25^{\text {th }}$ and $75^{\text {th }}$ percentiles, respectively. Whiskers show the maximum or minimum values while empty and filled circles represent the individual samples and outliers, respectively.

\section{DATA AVAILABILITY}

Gene accession numbers of all the Arabidopsis genes analysed in this study are listed in Methods. Source Data related to Fig 2b, c; Fig 3a, b, d; ED Fig 3d; ED Fig 4a, c, d; ED Fig 6a, b, c, d, e; ED Fig 7b, f, g; ED Fig 8a, b, c, d, e; and ED Fig $9 f$ are provided with the paper. All lines and other data supporting the findings of this study are available from the corresponding author upon request. 
572 Extended Data Figure 1 | Ontogeny of different cell types after activation of secondary growth in Arabidopsis 573 root. a, Bright field microscopy of root cross sections of an early phloem marker (PEAR1), a marker of 574 differentiating phloem expressed in phloem-companion cells (APL), an early xylem marker (AtHB8) and a marker 575 specific for differentiating xylem vessels (VND6) at different times after secondary growth activation. b, 576 Histological analysis of different cell types found in 14- and 30-day old root expressing a phloem differentiation marker PAPL:GUS (top) and wild-type (bottom). Cambium (red) typically consists of 2-4 cells (14 days) or 7-9 cells 578 (30 days). It is located between fully differentiated phloem elements (purple) and xylem vessels (blue), and is 579 characterized by frequent cell divisions detected histologically as a recently made thin cell wall. Xylem- and 580 phloem-parenchyma cells are quiescent cells located between xylem vessels or rarely dividing cells located between differentiated phloem cells, respectively. Data shown are representative of at least three independent experiments. Scale bars represent $50 \mu \mathrm{m}$.

583

Extended Data Figure 2 | A novel lineage tracing tool for developmental studies in plants. a, A schematic representation of two clonal activation systems. CRE recombinase was fused to the $\mathrm{N}$-terminal part of CYCB1;1 ${ }^{57}$ 586 to make CYCB1;1-CRE stable only in meristematic cells transiting between the early G2 and M phases of the cell cycle. Heat-shock induction of pHS:CYCB1;1-CRE causes recombination of two loxP sites which activates expression of 35S:GUS (i) or 35S:GAL4 (ii), respectively. In the second step, the latter transactivates pUAS:erGFP (iii) and, if desired, another gene of interest cloned under pUAS in a third construct (iv). b, Confocal microscopy of the root (RAM) and shoot (SAM - dashed line, top view) apical meristems, lateral root primordium (LRP) and procambium (PC) containing GFP clones $15 \mathrm{~h}$ after induction in 5-day old seedlings harbouring $\mathrm{pHS}$ :CYCB1;1-CRE and $p C B 1^{32}$ constructs. Because of the instability provided by the CYCB1;1-CRE fusion and the apparent nonsynchronicity of the cell cycle, a high frequency of single-cell descent clones was obtained almost exclusively in the dividing cells in the apical and lateral meristems. c, Examples of the three different GUS sector categories: "basic sectors" originate from a single cell clone induced before the activation of secondary growth (red, 75\%),

"large sectors" originate from a cluster of clones simultaneously induced in several neighbouring cells (black, 19\%), "half sectors" originate from a single cell clone induced after the activation of secondary growth (purple, $6 \%$ ). A total of 176 sectors were analysed. $\mathbf{d}$, A schematic representation of vascular cambium (red) and phellogen 599 (blue) ontogeny. Under normal conditions (top) both meristems originate from xylem pole pericycle (XPP) cells. 600 When roots are pre-treated with cytokinin (CK), cells in the protoxylem position (black arrowheads) remain 601 procambial cells ${ }^{56}$, and are thus capable of contributing to secondary growth (bottom, 'CK'). In these 
602

603

604

605

606

607

608

609

610

611

612

613

614

615

616

617

618

619

620

621

622

623

624

625

626

627

628

629

630

631

632 experimental conditions, separation of vascular cambium and phellogen lineage occurs already during the primary growth, and xylem-connection remains the determining factor for the origin of vascular cambium. e, Examples of GUS clones $24 \mathrm{~h}$ after heat shock in cytokinin (BAP) pre-treated roots. Arrowheads, procambial cells in place of protoxylem; asterisks, pericycle cells which have lost their xylem connection. $\mathbf{f}$, Correlation of different GUS sector shapes with the presence (left) or absence (centre, right) of protoxylem (insets, arrowheads). The numbers represent the frequency of these sectors. $\mathbf{g}$, XPP cell fate map of BAP-pre-treated roots. XPP cells which lose their xylem connection behave like pericycle cells (light purple, compare with Fig. 1b); cells at the protoxylem position behave like xylem-procambial cells, making wide sectors (red, compare with Fig. 1b). In b, e, f, experiments were repeated at least two times with similar results. Scale bars represent $50 \mu \mathrm{m}$.

Extended Data Figure 3 | Transcriptional and mutant analyses of HD-ZIP III transcription factors. a, In situ hybridisation of sense- and anti-sense probes in 21-day old Col-0 cross sections showing HD-Zip IIls expression in the cambium. Insets are 2x magnifications of the cambial region of the same photo. $\mathbf{b}$, Confocal microscopy of cross section of 14-day old pAtHB8:erYFP marker line. Cell wall staining in grey. Red arrowheads, recent cell divisions in cambium. c, Bright field microscopy of primary and secondary vasculature shows patterning defects in higher order HD-ZIP III mutants 7 days and 10 days after germination. Col-0 and cna-2 phb-13 phv-11 show normal differentiation of the primary xylem axis and two phloem poles, whereas the majority of athb8-11 cna-2 phb-13 phv-11 roots did not have a fully differentiated xylem axis or showed a triarch pattern with three phloem poles. The fraction in upper left corner denotes the frequency of the presented phenotype. Asterisks, primary phloem poles; arrowheads, extremities of the primary xylem. $\mathbf{d}$, Confocal images of EdU-stained (green) nuclei in cna-2 phb-13 phv-11, athb8-11 cna-2 phb-13 phv-11 mutants and the miR165a overexpressor (top). miR165a was induced for 12 days (in 4-day old plants), and the mutants were 14 days old. Inhibition of HD-ZIP IIIs leads to cambium disorganisation (histograms, bottom). Even though AtHB8, CNA, PHB and PHV, and perhaps also REV are redundantly required for secondary xylem formation and proper cambium organisation, it is possible that individual members of the family have distinct function in the cambial stem cells and their organisers. Histograms of the relative distances (RD) of EdU-positive nuclei and secondary xylem vessels in the radial direction from the root midpoint (xylem axis: $x$ value $=0$, periderm: $x$ value $=1$, with the scale shown in green in the Col-0 panel). KLD indicates the mean Kullback-Leibler distances between EdU-positive nuclei and vessels. $n_{\mathrm{c}}=$ number of cross sections analysed; $n_{\mathrm{n}}=$ total number of nuclei. In $\mathbf{a}$ and $\mathbf{b}$, data shown are representative of two (a) or four (b) independent experiments. Scale bars represent $50 \mu \mathrm{m}$. 

III expression after miR165a induction for $24 \mathrm{~h}$ (in 9-day old plants). b, miR165a overexpressing plants grown on induction medium for 14 days (from germination onwards) resemble the quintuple HD-ZIP IIIs mutant ${ }^{8}$. c, Quantification of secondary xylem vessel formation after miR165a induction for 2 days (in 8-day old plants). Phenotypic changes on secondary vessel number start to appear after 2 days of the induction. A two-tailed Wilcoxon test ( $n=25$ independent roots). d, qRT-PCR analysis for marker gene expression after 2-day miR165a induction (in 8-day old plants). e, Bright field microscopy of miR165a overexpressor double stained with Safranin and Alcian blue to highlight secondary phloem (insets) after miR165a induction for 12 days (in 4-day old plants). Red dots, secondary phloem; asterisk, primary phloem; arrowheads, extremities of the primary xylem. $\mathbf{a}$ and $\mathbf{d}$ one-tailed t-test. Data are from three experiments ( $n=10$ roots per treatment). In $\mathbf{b}$ and $\mathbf{e}$, the fraction denotes the frequency of the presented phenotype. Box-and-whisker plots show median and interquartile range; whiskers show range, dots outliers. Scale bars represent $50 \mu \mathrm{m}$. sectors obtained after clone induction in 15- (a) and 30-day (b-d) old plants harbouring pHS:d-Box-CRE and 35S:Iox-GUS constructs (see Extended Data Fig. 2a). a, b, Middle panels are lateral view of roots with GUS sectors before sectioning. $\mathbf{c}$, $\mathbf{d}$, Lineage tracing in 30-day old cambium revealed that bifacial stem cell is present even in wide cambium where 7 - 9 cell layers could be identified histologically (see Extended Data Fig. 1b). Quantification was performed as in Fig. 1d. e-h, Lineage tracing in ANT expression domain. e, A schematic representation of clonal activation system to induce GUS clones in ANT expression domain. After induction, CRE-directed recombination leads to clonal activation of GUS reporter. $\mathbf{f}$, GUS sectors obtained after clone induction in 17-day old plants harbouring pANT:XVE >>CRE and 35S:lox-GUS. Inset is a 2,5x zoom of the cambial zone of the same picture. $\mathbf{g}, \mathbf{h}$, Lineage tracing in active cambium showed $A N T$ as a stem cell marker. Six days after induction (in 17-day old plants), growing GUS sectors had either xylem fate (green) or phloem fate (yellow), but the majority (76\%) had cambial fate (red) based on the position of thinnest cell wall (arrow) in radial direction, representing the most recent periclinal cell division in cambium ( $y$ value $=0$ ). $\mathbf{d}, \mathbf{h}$, Each bar represents the size and position of an individual sector $(n=131)$; bars with extensions denote sectors beginning/ending with a xylem vessel. $\mathbf{a}, \mathbf{b}$, f, Red arrows, sectors originating from cambium stem cells; yellow arrow, sectors on the phloem side; green arrow, sectors on the xylem side; brown arrow, an example of sectors excluded from the quantification analysis. GUS staining is weak or absent in differentiated xylem vessels. Data represents sectors collected across at least 

expression after AtHB8d induction for $24 \mathrm{~h}$ (in 9-day old plants). b, Confocal images of EdU staining reveal a centrifugal shift of the cell division domain after 2-day AtHB8d induction (in 8-day old plants). Asterisks mark cambium. Histograms showing the relative distances (RD) of EdU-positive nuclei in the radial direction from the root midpoint (xylem axis: $\mathrm{x}$ value $=0$, periderm: $\mathrm{x}$ value $=1$ ), as depicted in Mock. $n_{\mathrm{n}}$, total nuclei number. $\mathbf{c}$, Quantification of secondary xylem vessel formation after AtHB8d induction for 2 days (in 8-day old plants). d, Confocal microscopy of fluorescent markers after 2-day AtHB8d induction (in 8-day old plants). Cell wall staining in grey. Dashed lines, borders of the secondary tissues. Graph is quantification of the relative width of marker expression domains. e, Bright field microscopy of roots after AtHB8 induction for 4 days (in 8-day old plants) and quantification of secondary xylem vessel numbers. Red dots, secondary xylem vessels; yellow dashed line, cambium position in mock-treated roots. $\mathbf{a}$ and $\mathbf{d}$, One-tailed t-test. Data are from three experiments $(n=10$ roots per treatment). $\mathbf{c}$ and e, Two-tailed Wilcoxon test. $n$, independent roots. Box-and-whisker plots show median and interquartile range; whiskers show range, dots outliers. Scale bars represent $50 \mu \mathrm{m}$.

679

Extended Data Figure 7 |Auxin signalling and ARF reporters. a, Bright field microscopy of DR5rev:GUS cross 681 section of a 14-day old root. b, Confocal microscopy of DR5V2:nGFP (heatmap) root cross sections. Nuclear 682 fluorescence signal (examples of ROIs are indicated with a circle) was quantified in three cell layers from the 683 most recent cell division (arrowheads) towards xylem (positions -1, -2, -3) and phloem (positions 1, 2, 3). Fourteen cross sections were analysed; $n_{n}=$ total number of nuclei. c, Bright field microscopy of 17-day old roots expressing class A ARF GUS markers. d, Confocal microscopy of 14-day old plant expressing pAtHB8:erRFP and 686 pMP:erYFP. e, Confocal and bright field microscopy of pMP:erYFP and DRrev:GUS marker width after 2-day AtHB8d induction in 8-day old plants. Nuclear localization of AtHB8d-YFP is shown in Extended Data Fig. 6d. pMP:erYFP roots were stained with calcofluor (grey). The fraction in bottom left corner denotes the frequency of the presented phenotype. White bars, width of the marker domain. Dashed lines, borders of the secondary tissues. f, qRT-PCR analysis of MP expression after 2-day miR165a induction in 8-day old plants. Data are from three experiments ( $n=10$ roots per treatment). $\mathbf{g}$, Quantification of the relative width of marker expression 692 domains after pANT:XVE>>AtHB8d-YFP induction shown in (e) (right panels). Sample size for each group is equal to denominator of the fraction indicated in (e). $\mathbf{b}, \mathbf{f}$ and $\mathbf{g}$ one-tailed t-test for the comparison between different positions (b) or between mock and induced sample (f, $\mathbf{g})$. Box-and-whisker plots show median and interquartile 695 range; whiskers show range, dots outliers (g) or individual data points (b). a, c, d, Representative images of at 
696

697

698

699

700

701

702

703

704

705

706

707

708

709

710

711

712

713

714

715

716

717

718

719

720

721

722

723

724

725

726

727

least three independent observations. $\mathbf{b}, \mathbf{d}$ and $\mathbf{e}$ cell wall staining in grey. $\mathrm{p}$, phloem; $\mathbf{x}, \mathbf{x y l e m}$. Scale bars represent $50 \mu \mathrm{m}$.

Extended Data Figure 8 | Phenotypic characterization inducible amiMP and axr3-1 lines. a, The arf7, 19 double mutant shows no difference in secondary xylem production when compared to wild-type (Col-0) and analysed 14 days after germination, ( $n_{\text {col- } 0}=17$ and $n_{\text {arf7,19 }}=15$ independent roots). b, Quantification of secondary xylem vessel formation after amiMP induction for 7 days (in 5-day old plants) in wild-type ( $n_{\text {col-o }}=7$ independent roots) and $\operatorname{arf7,19}\left(n_{\text {arf7,19 }}=34\right.$ independent roots) plants analysed $15 \mathrm{~mm}$ below the root-hypocotyl junction. The reduction in xylem vessel formation was less apparent when amiMP was induced in the wild-type background, indicating that ARF7 and/or ARF19 are also required for this process. c, Plots of regressions between MP transcript levels and vasculature diameter (left), secondary xylem vessel formation (centre) and cell divisions (right) analysed $5 \mathrm{~mm}$ (top) and $15 \mathrm{~mm}$ (bottom) below the root hypocotyl junction. Each point represents an individual T1 transformant or $\operatorname{arf7,19}$ plant $\left(n_{\text {amiMP }}=33 ; n_{\text {arf, 19 }}=6\right)$ grown for 5 days on a basta selection plate or $1 / 2 \mathrm{GM}$ and then induced for 7 days. Since the residuals were not normal, a one-way Wilcoxon test for the similarity of the means of the $\operatorname{arf7,19}$ and amiMP groups on MP transcription level was performed. A negligible $P$-value of $3.065 \times 10^{-7}$ confirms that the $\operatorname{arf7,19}$ mean is significantly greater than the amiMP mean. In order to assess the linear relationship between MP transcript levels and the observed phenotypes, we performed regression analyses. The plotted blue line indicates the fitted regression model to both groups combined, and the $P$-value indicating the goodness of this fit is plotted on the top of each graph, respectively. $d$, qRT-PCR analysis of HD-ZIP IIls behaviour after 24 and 72h induction of axr3-1 (in 8-day old plants). Data are from four experiments ( $n=16$ roots per treatment). e, Bright field microscopy and secondary xylem vessel quantification (bottom) of roots after axr3-1 induction for 48 and $72 \mathrm{~h}$ (in 8-day old plants). Insets are $1.5 \times$ magnifications of the cambial region of the same photo. Arrowhead; recent cell division. Sample size for each group is equal to denominator of the fraction indicated in upper panels. f, Bright field microscopy of roots after axr3-1 induction for 7 days (in 5-day old plants). Box-and-whisker plots show median and interquartile range; whiskers show range, dots outliers. $\mathbf{a}$ and $\mathbf{b}$ Twotailed Wilcoxon test. $\mathbf{d}, \mathbf{e}$, One-tailed t-test. In $\mathbf{e}, \mathbf{f}$, the fraction in upper left corner denotes the frequency of the presented phenotype. Scale bars represent $50 \mu \mathrm{m}$.

Extended Data Figure 9 | Clonal activation in the S17 expression domain. a, A schematic representation of the clonal activation system to induce clones in phloem pole pericycle cells and surrounding tissue. After induction, CRE-directed recombination leads to clonal activation of gene-of-interest (GOI: AtHB8d; MPA; gVND6) tagged with a reporter. $\mathbf{b}$, Bright field microscopy of GUS sectors in S17 position analysed 1 day after clone induction (in 
728

729

730

731

732

733

734

735

736

737

738

739

740

741

742

743

744

745

746

747

748

749

750

751

752

753

754

755

756

757

758

759

5-day old plants). Induction of "large" sectors consisting of several single cell GUS clones in the root tip region recapitulated previously described expression pattern of the S17 promoter being specific to phloem-pole pericycle cells (bottom left; Lee et al.). Higher up in the root where secondary growth has already started, GUS clones were obtained in primary phloem and surrounding tissue (right panels) suggesting that after secondary growth activation the S17 expression domain enlarges centripetally. Experiment was repeated twice with similar results. c, None of 21 35S:Iox-CATHB8d-GUS transformants in pS17:XVE>>CRE background showed ectopic vessel formation. Confocal (left) and bright field microscopy (right) analysis of AtHB8-GUS clones in a cambial marker background. Marker expression was analysed 2 days after clone induction (in 5-day old plants). The bright field and confocal images are from different roots. $n=$ number of entire roots screened for ectopic marker expression. d, Confocal analysis of AtHB8d-GUS clonal activation lines performed 7 days after AtHB8-GUS clone induction (in 5-day old plants), with calcofluor staining in grey. $n=$ number of entire independent roots screened for ectopic vessel formation. e, f, MPA- and gVND6-induced ectopic vessels show different levels of periclinal cell division induction in neighbouring cells. Bright field microscopy of root cross sections stained with toluidine blue showing ectopic vessels with periclinal cell division in neighbouring cells analysed 7 days after induction (in 5-day old plants). Yellow dots, ectopic xylem vessel; red dots, cell which have recently undergone periclinal cell division. $n$ = number of xylem vessels examined for each category (0-7 neighbouring cells dividing periclinally). Quantification in (f). Data were collected across 16 independent roots for each genotype. $\mathbf{g}$, Confocal analysis of marker expression within gVND6-YFP clones (top) and around $g$ VND6-YFP-induced ectopic vessels (bottom). The fraction in upper right corner denotes the frequency of the presented phenotype. Cell walls were stained with calcofluor (grey). Insets show merged YFP and RFP channels except for insets of WOX4 showing only YFP channel. Scale bars represent $50 \mu \mathrm{m}$.

Extended Data Figure 10 | Model describing the dynamic nature of vascular cambium. a, When the organiser cells differentiates into a xylem vessel, a new organiser is formed in adjacent cambial stem cell. Stem cells and their xylem side daughters dynamically gain organiser cell gene expression as they mature towards xylem. Only the events leading to xylem production are illustrated in the model: mechanism for phloem production from the bifacial stem cell is unknown and thus not presented in the scheme. Density of the dots corresponds to the level of signalling components shown in (b). $\mathbf{b}$, A model presenting a signalling framework defining the stem cell organiser of vascular cambium. Question marks refer to unknown factors that cell non-autonomously promote stem cell or phloem identity. These mobile, stem cell promoting factors could for example be cytokinins, which during the primary development move from primary xylem precursor cells, marked with high auxin signalling, to promote cell division in the neighbouring procambial cells ${ }^{58-60}$. Supporting this scenario, cytokinins are required 
760 for cambial cell proliferation during the secondary development ${ }^{61}$. Alternatively, WOX4 or other transcription 761 factors expressed in the organiser could move to the adjacent cell to promote stem cell identity, similar to WUS 762 and WOX5 in the apical meristems².

763 
Figure 1

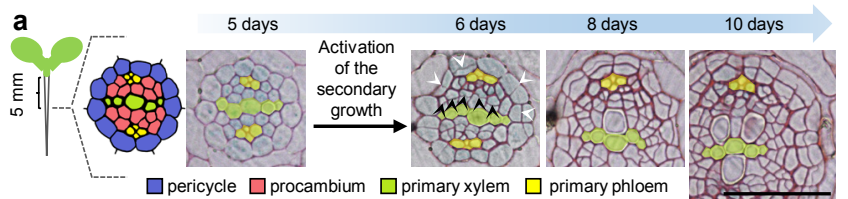

$\bigotimes$ first divisions in procambium $\boldsymbol{\Lambda}$ first divisions in pericycle

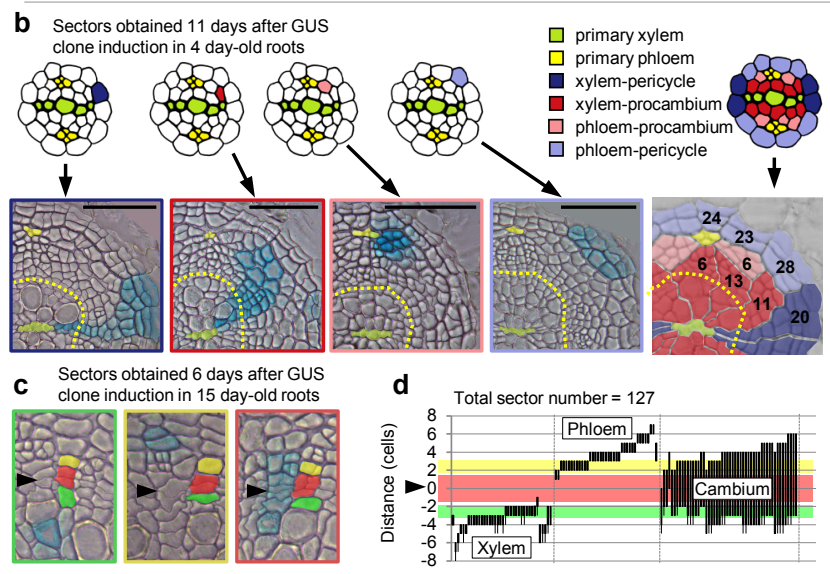

Figure 1 | Lineage tracing revealed the origin of the vascular cambium stem cells. a, The activation of secondary root growth is characterised by the initiation of periclinal cell divisions in procambial cells (black arrowheads), followed by divisions in pericycle (white arrowheads), leading to the formation of vascular cambium and phellogen. Cell divisions are marked only in upper half of the cross section. $\mathbf{b}$, Lineage tracing initiated from the primary vasculature (top) showed the importance of an initial xylem connection for the ontogeny of the cambium (yellow dashed line). The bottom panel shows the four major types of clonal sectors obtained. Frames are coloured to match with the corresponding cell lineages in the top panels. The numbers in the fate map (bottom right) refer to the observed number of the sectors. c, $\mathbf{d}$, Lineage tracing in active cambium revealed a common stem cell for xylem and phloem. GUS sectors (black bars) plotted based on the position of thinnest cell wall (arrow) in each radial file, representing the most recent periclinal cell division in cambium ( $y$ value $=0$ ). Scale bars represent $50 \mu \mathrm{m}$. All experiments were repeated four times. 
Figure 2

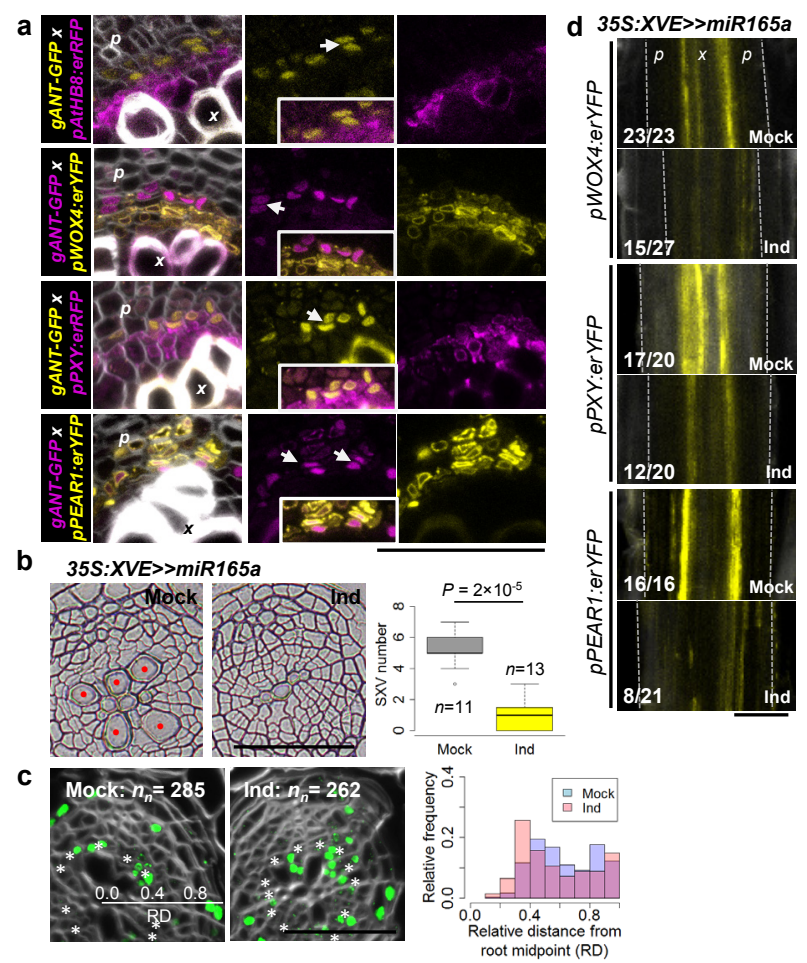

Figure 2 | HD-ZIP Ills determine the organiser of the vascular cambium. a, Confocal microscopy of cross sections of 14-day old ANT double markers. White arrows indicate pairs of nuclei in recently dividing cells. Insets, merged reporter channels. b. Bright field microscopy of root after miR165a induction (Ind) for 6 days (in 5-day old plants). Red dots, secondary xylem vessels (SXV); quantification presented on the right. Box-and-whisker plot shows median and interquartile range; whiskers show range, dots outliers. Two-tailed Wilcoxon test. $n$, independent roots. $c$, Confocal images of EdU staining reveal a centripetal shift of the cell division domain after 2-day miR165a induction (in 8-day old plants). Asterisks mark cambium. Histograms showing the relative distances (RD) of EdU-positive nuclei in the radial direction from the root midpoint (xylem axis: $x$ value $=0$, periderm: $x$ value $=1$ ), as depicted in Mock. $n_{n}$, total number of nuclei. $\mathbf{d}$, Confocal microscopy of fluorescent markers after 2 day miR165a induction (in 8-day old plants). Dashed lines, borders of the secondary tissues. The numbers in the bottom left corner of subpanels represent the frequency of the observed phenotype. a, $\mathbf{c}$, d, Cell wall staining in grey. p, phloem; $x$, xylem. All experiments were repeated at least three times. Scale bars represent $50 \mu \mathrm{m}$. 
Figure 3
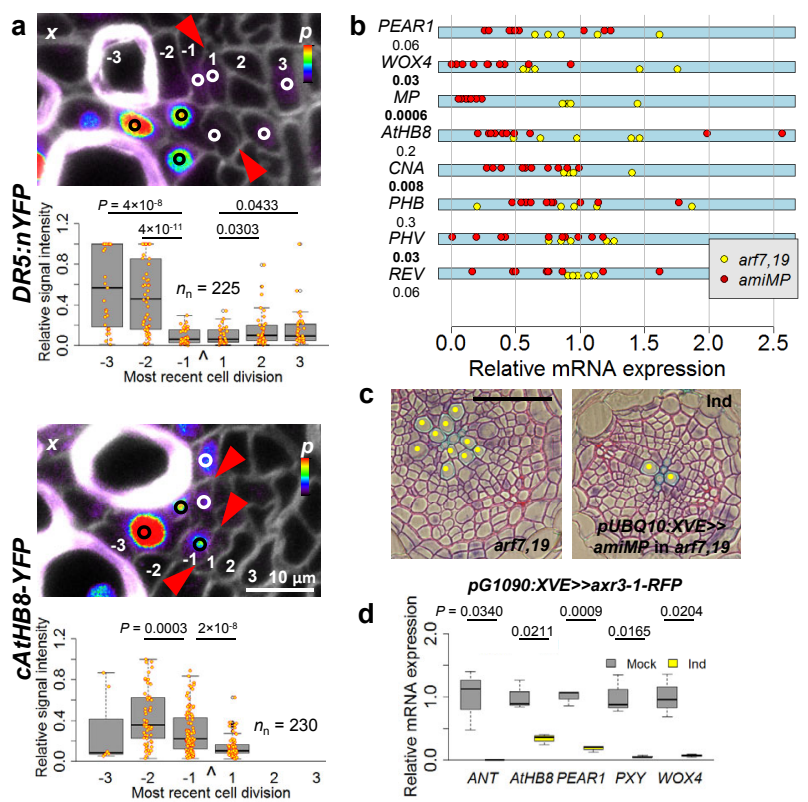

Figure 3 | Auxin signalling is required for xylem differentiation and organizer identity. a, Confocal microscopy of DR5:nYFP and CAtHB8-YFP (heatmap) root cross sections. Nuclear fluorescence signal (circles indicate examples of ROIs) was quantified in three cell layers from the most recent cell division (arrowheads) towards xylem (positions $-1,-2,-3$ ) and phloem (positions $1,2,3$ ). $n_{n}=$ total number of nuclei. Cell wall staining in grey. $p$, phloem; $x$, xylem. $\mathbf{b}$, qRT-PCR analysis of marker gene expression in amiM P T1 transformants with the lowest MP transcript levels. Each point represents an individual amiM P T1 transformant $(n=10)$ or individual arf7,19 plant $(n=5)$ of the same age. The $p$-values of a one-sided t-test comparing amiM $P$ to arf7,19 controls are provided under each gene name, and significant values are in bold-face. c, Bright field microscopy of pUBQ10:XVE >>amiM P transformants in the arf7,19 background induced for 7 days (in 5-day old plants). Yellow dots, secondary xylem vessels. Experiment was repeated three times with similar results. $\mathbf{d}$, qRT-PCR analysis of marker gene expression in inducible axr3-1 after $24 \mathrm{~h}$ induction (in 8-day old plants). Data are from three experiments ( $\mathrm{n}=16$ roots per treatment). In $\mathrm{a}$ and d, box-and-whisker plots show median and interquartile range; whiskers show range. One-tailed t-test. In a, dots represents independent data points. Scale bars represent $50 \mu \mathrm{m}$. 
Figure 4

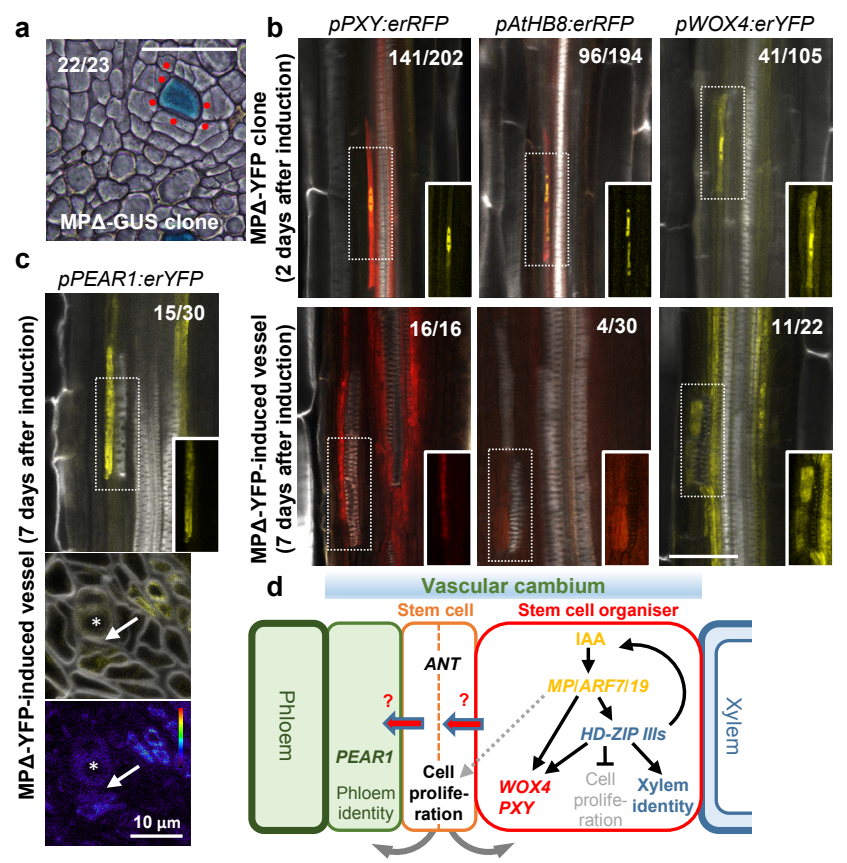

Figure 4 | MP is sufficient for the formation of an ectopic organiser. a, Bright field micrograph of an MPA-GUS clone with periclinal cell divisions induced cell non-autonomously. Red dots, GUSnegative cells dividing periclinally around the MPA-GUS clone. $\mathbf{b}$, Confocal analysis of marker expression within MPA-YFP clones (top) and around MPA-YFP-induced ectopic vessels (bottom). Cell wall staining in grey. Insets show YFP channel except for insets of PXY and AtHB8 in bottom panels showing RFP channel. c, PEAR1 is induced a few cell layers from MPA-induced ectopic vessels (asterisk). An arrow indicates a gap between ectopic vessel and PEAR1 expression. $\mathbf{d}, \mathrm{A}$ model illustrating how a local auxin maximum and the downstream components define an organiser which positions the stem cell in the adjacent cell. Curved arrows represent bidirectional cell proliferation from the bifacial stem cell. The numbers in the left corner of subpanels represent the frequency of the observed phenotype. Scale bars represent $50 \mu \mathrm{m}$ unless indicated otherwise. 


\section{Extended Data Figure 1}

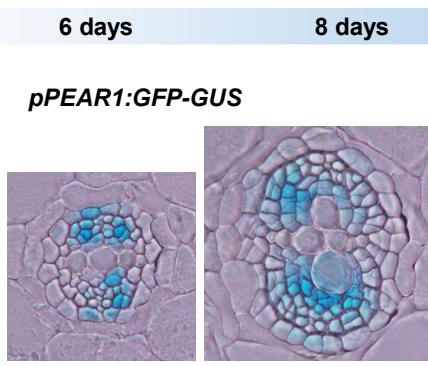

pAPL:GUS
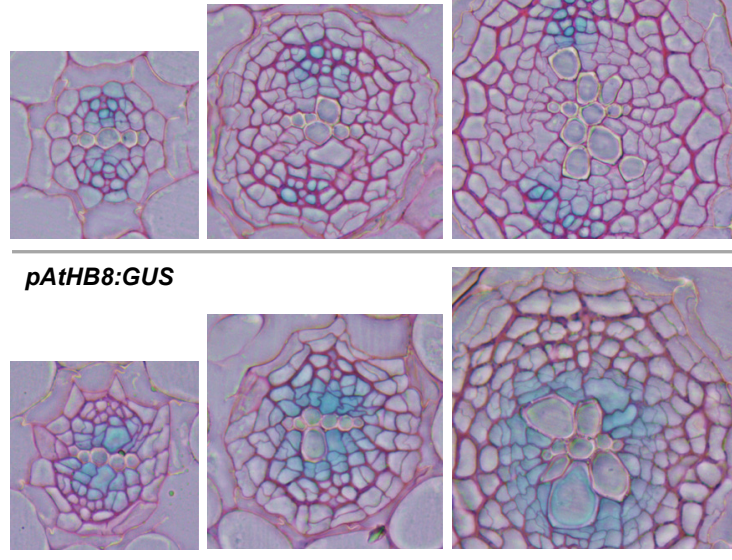

\section{gVND6-GUS}

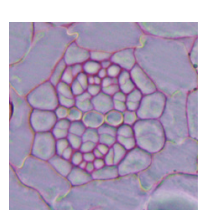

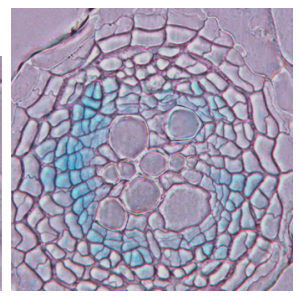
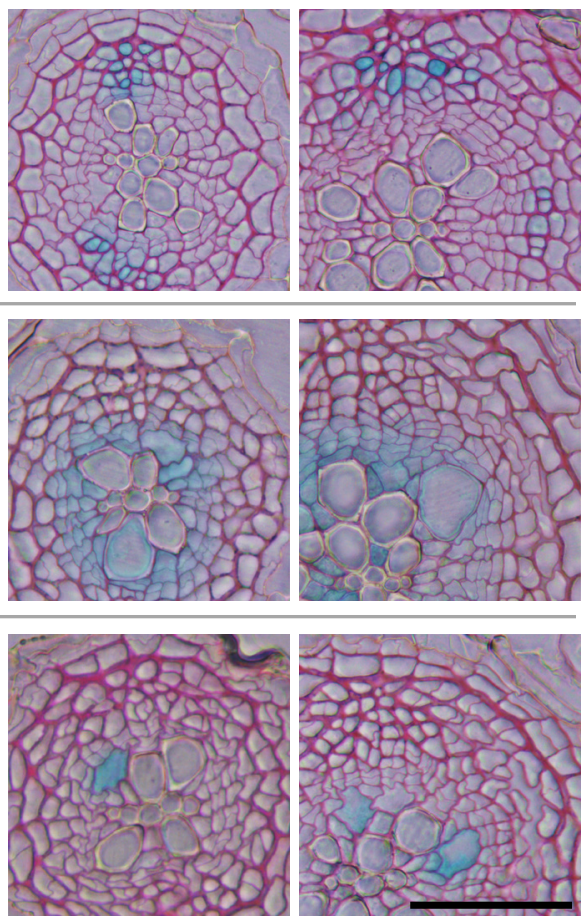

14 days
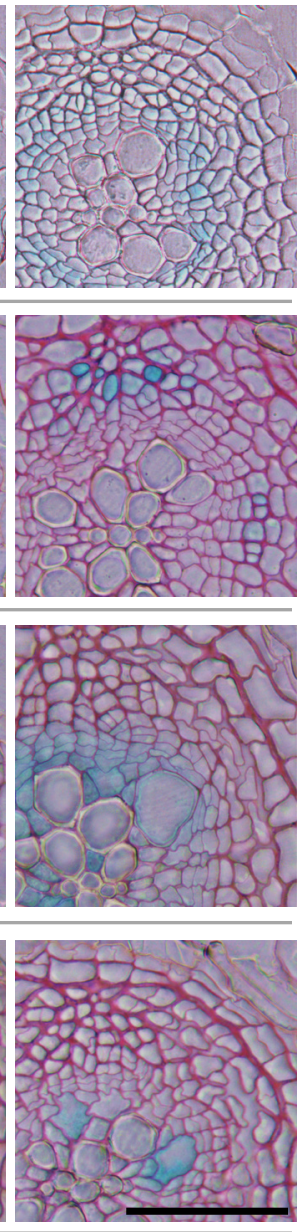

b
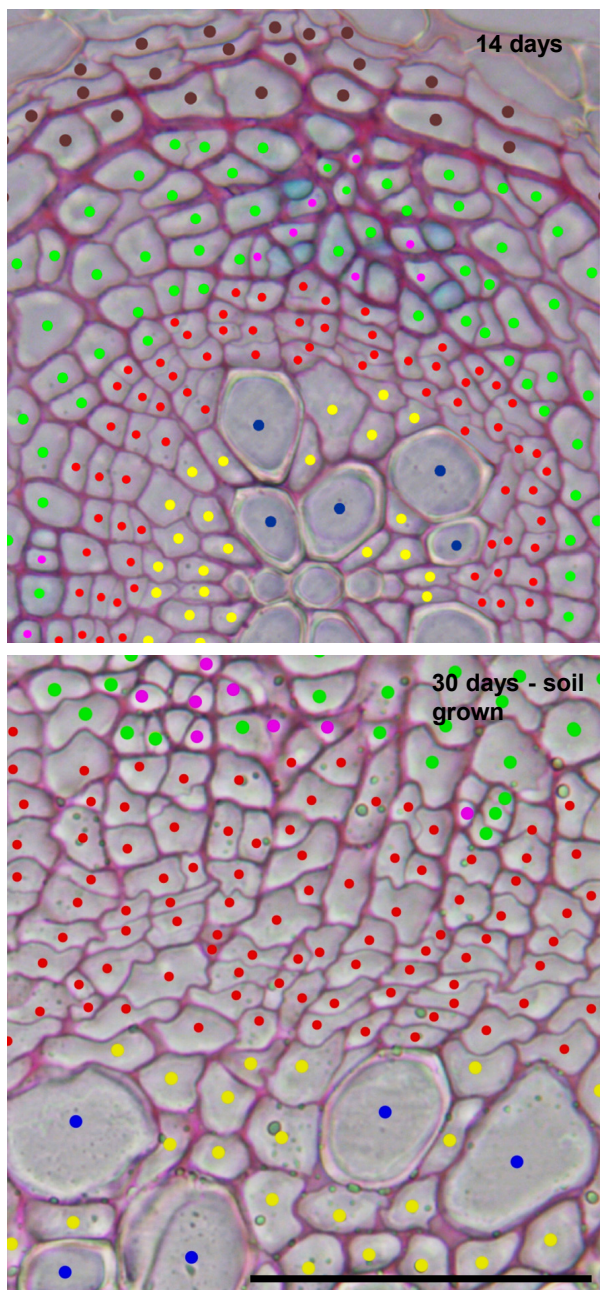

$\square$ phloem parenchyma

secondary xylem vessels

$\square$ vascular cambium

$\square$ xylem parenchyma cells

$\square$ periderm (phelloderm

$\square$ phloem sieve elements and neighboring companion cells (not marked) 


\section{Extended Data Figure 2}
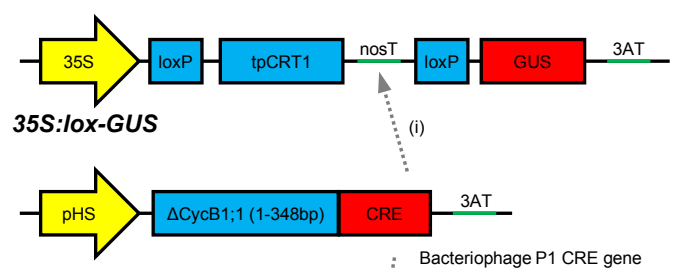

pHS:d-Box-CRE

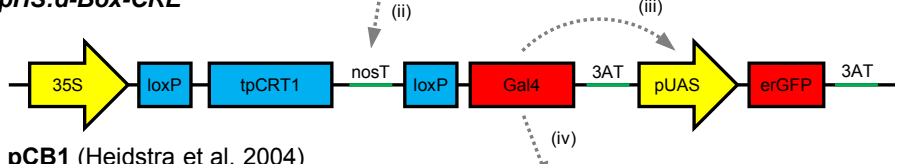

pCB1 (Heidstra et al. 2004)

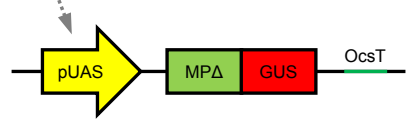

pUAS:MPA-GUS

C

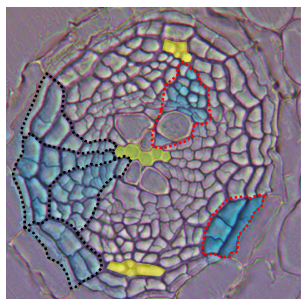

$\square$ primary phloem $\square$ primary xylem
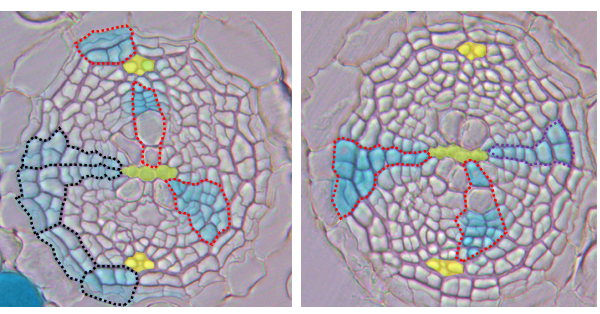

e
(-) protoxylem

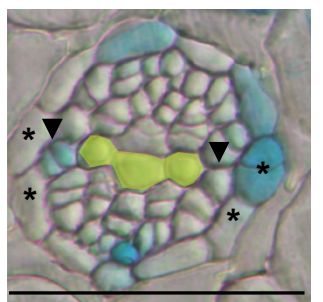

(+) protoxylem

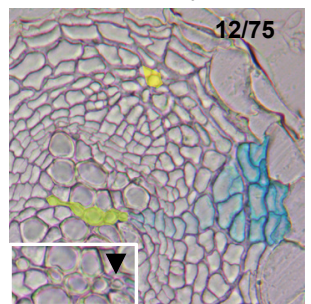

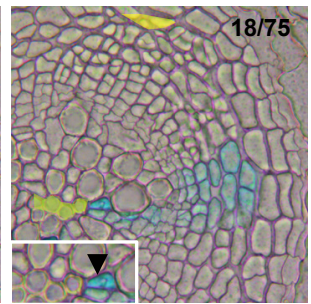

b

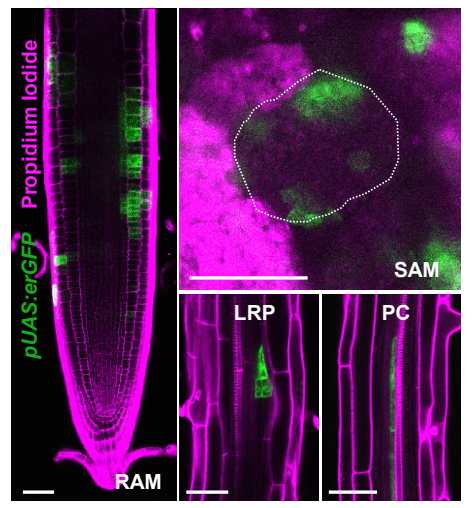

d 5 days

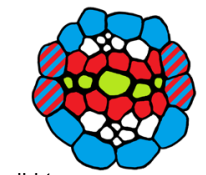

wild-type

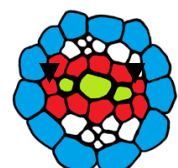

CK (BAP)

pre-treatment

(-) protoxylem
14 days

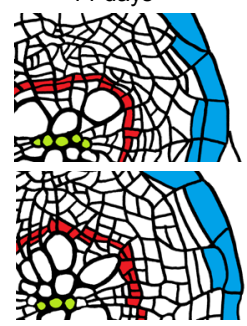

$\square$ primary xylem

$\square$ vascular cambium lineage

$\square$ phellogen lineage

$\square$ lineage for both, vascular g cambium and phellogen
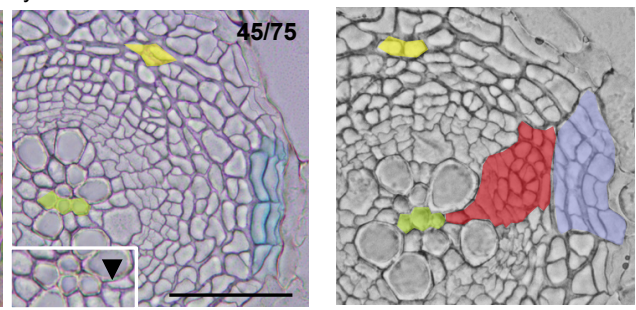


\section{Extended Data Figure 3}

a

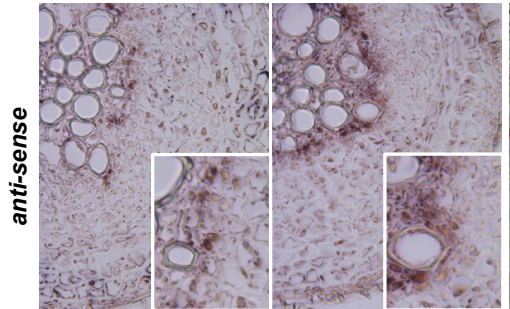

AtHB8

CNA

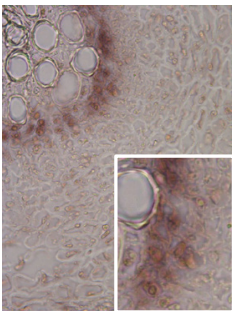

PHB

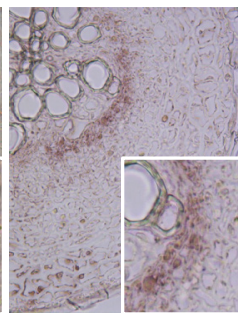

PHV
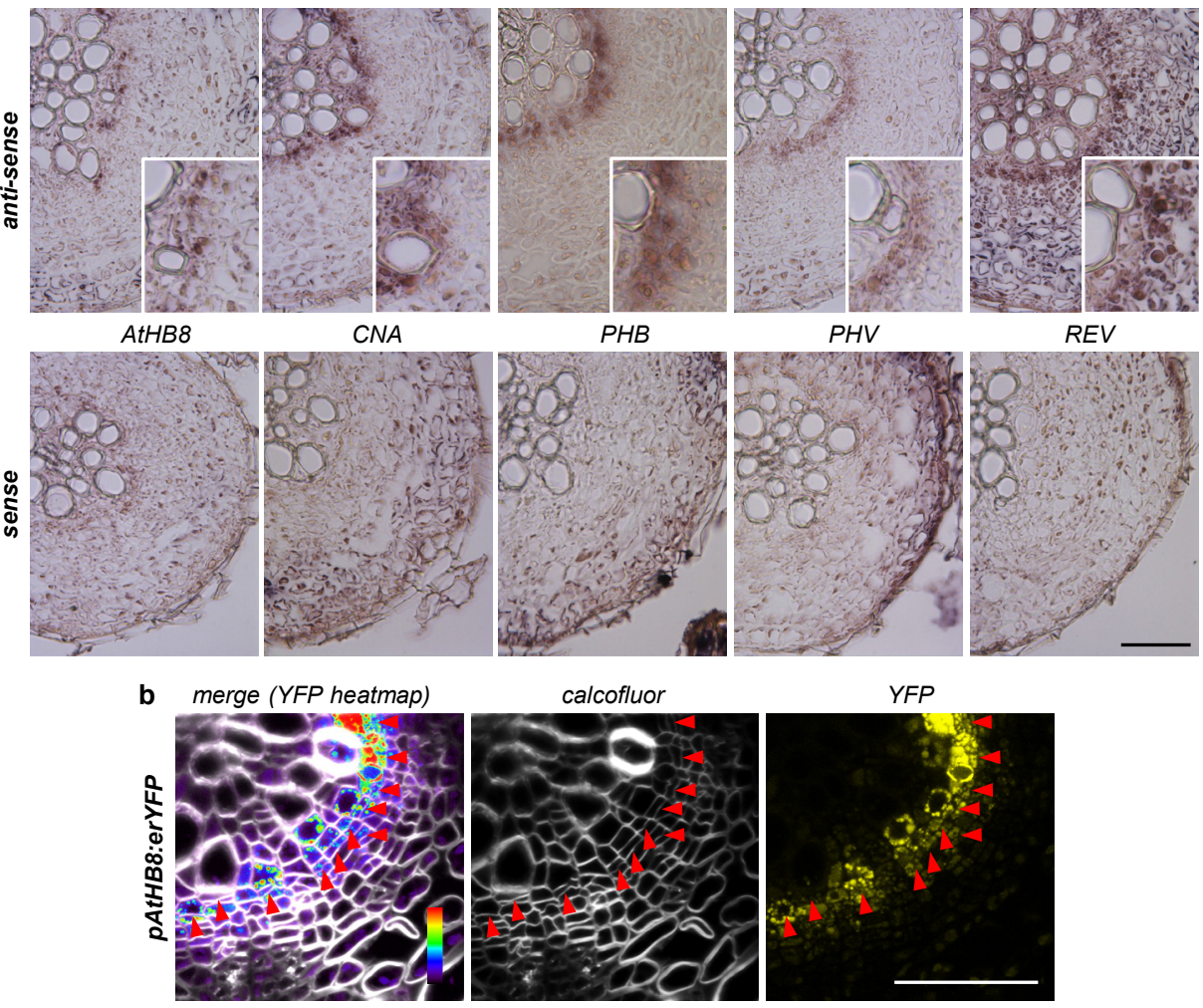

c

cna-2 phb-13 phv-11

athb8-11 cna-2 phb-13 phv-11
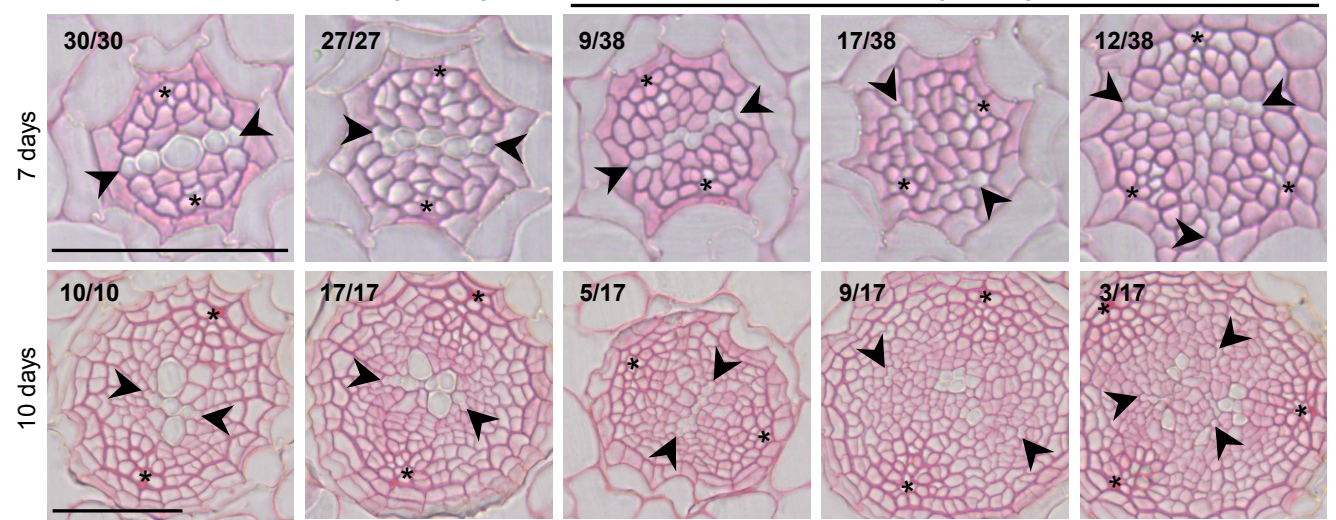

d
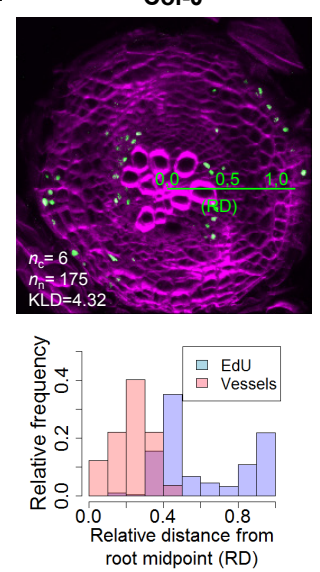

cna-2 phb-13 phv-11
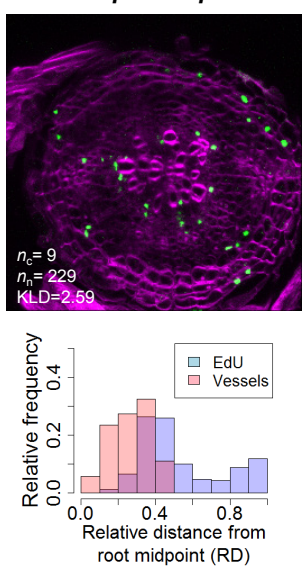

athb8-11 cna-2

phb-13 phv-11
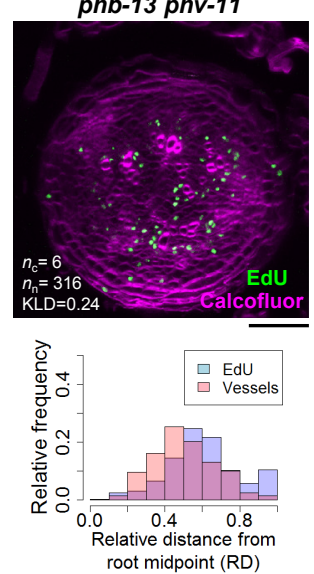
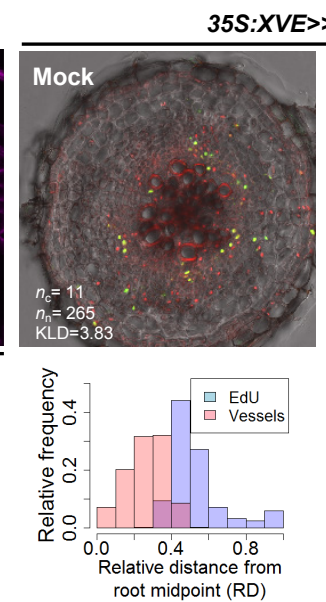
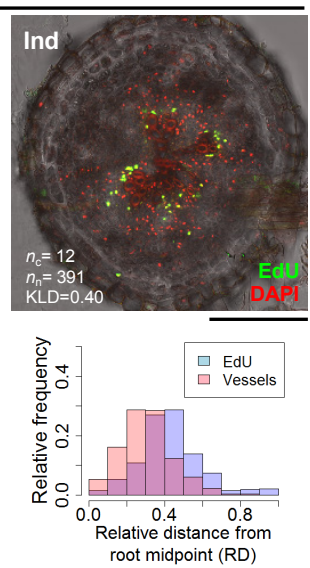


\section{Extended Data Figure 4}

a

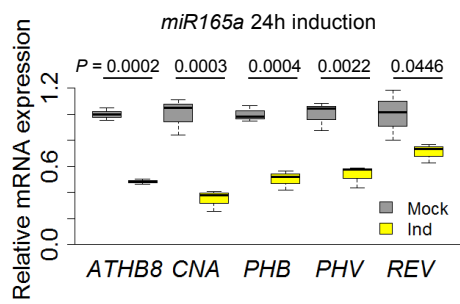

d

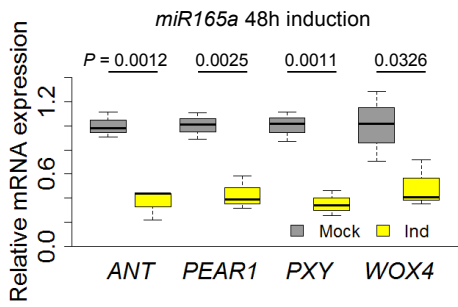

b

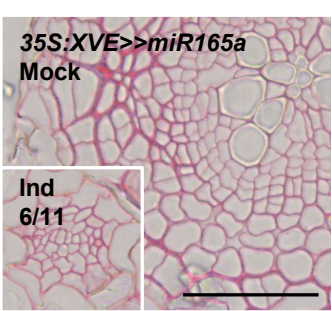

C

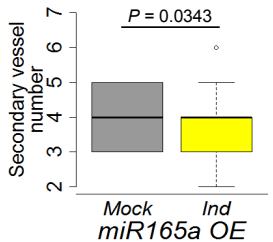

e

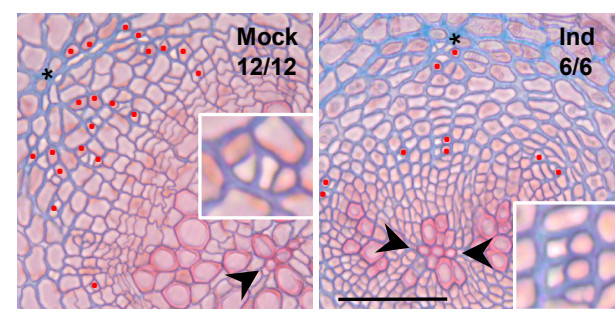




\section{Extended Data Figure 5}

a
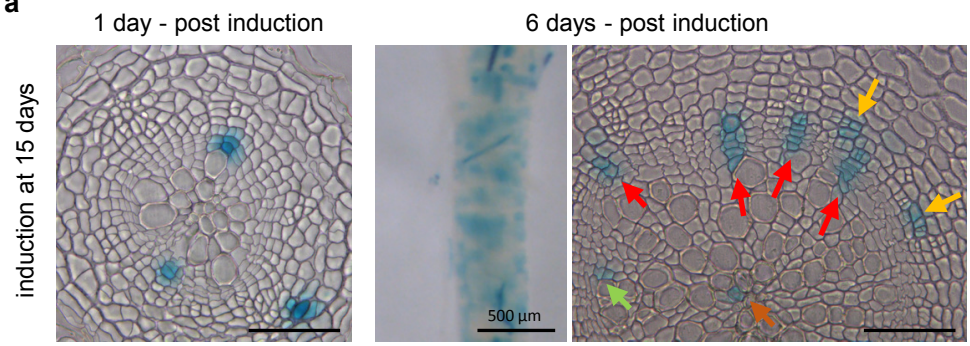

b 1 day - post induction

8 days - post induction
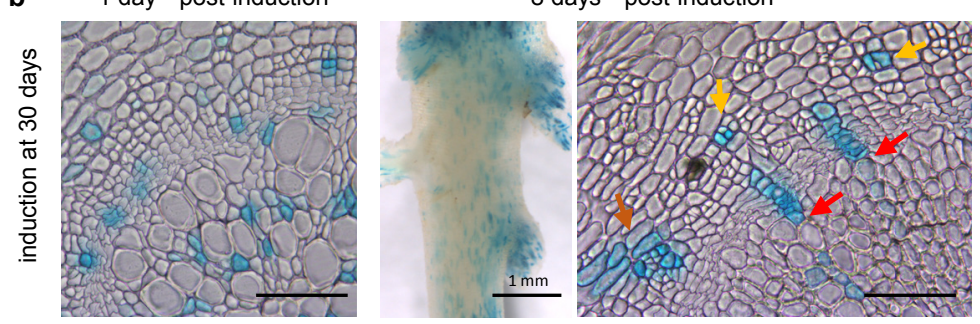

c

8 days - post induction
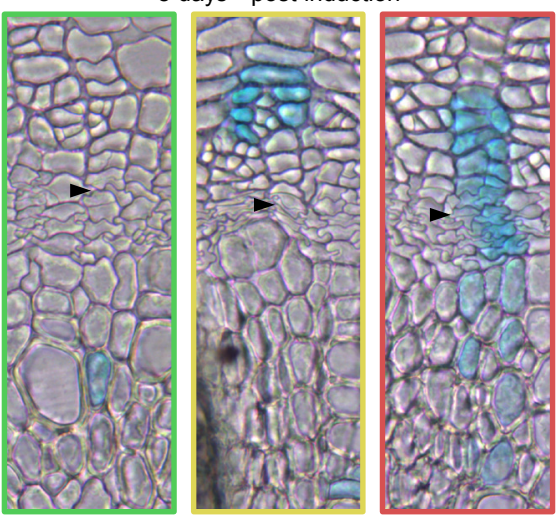

d

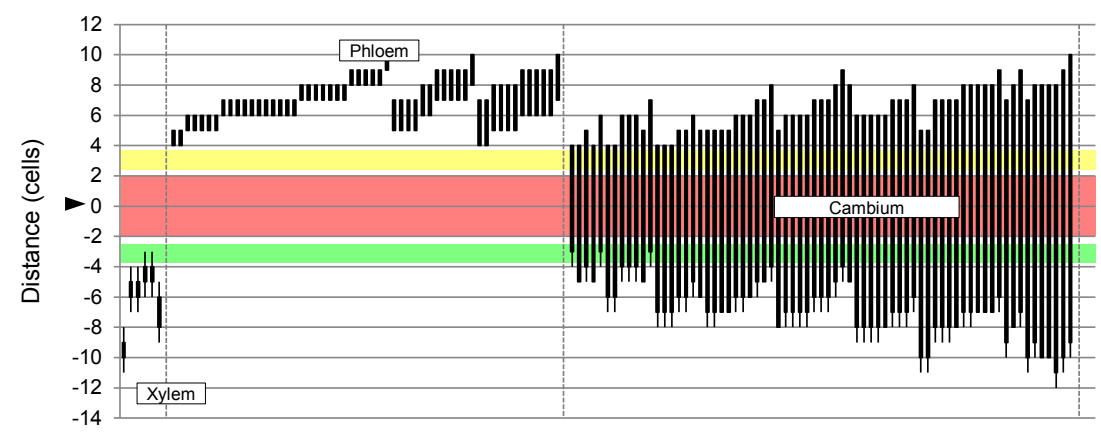

e

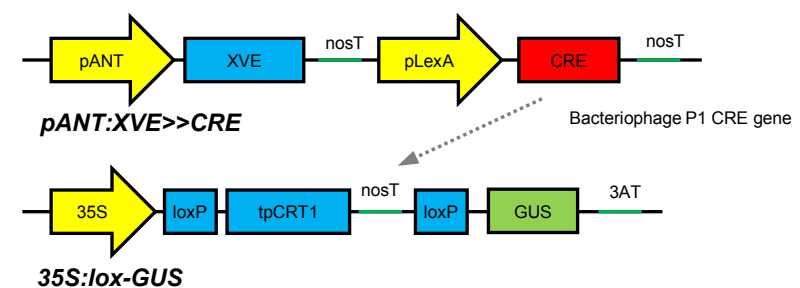

f

1 day - post induction

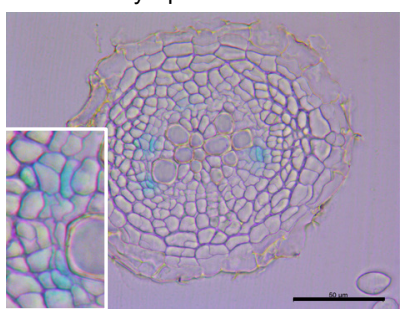

6 days - post induction

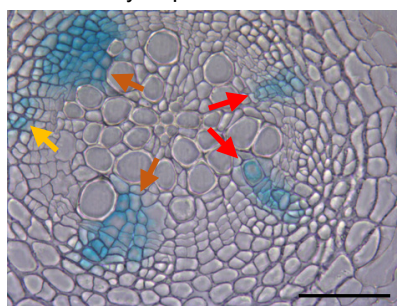

g

6 days - post induction

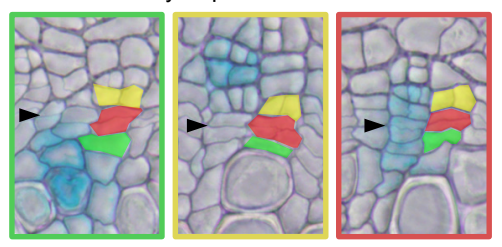

h

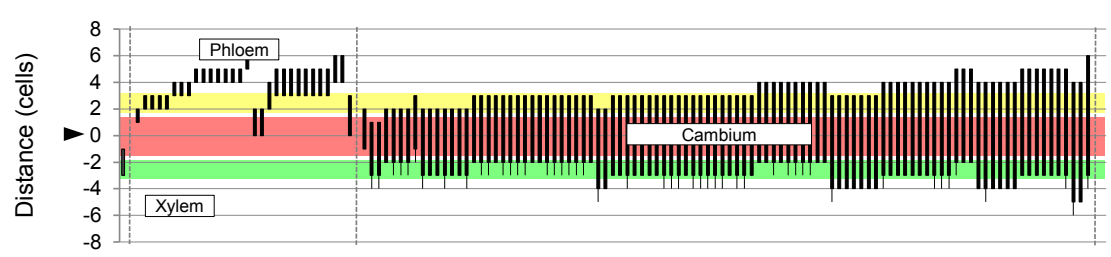




\section{Extended Data Figure 6}

a

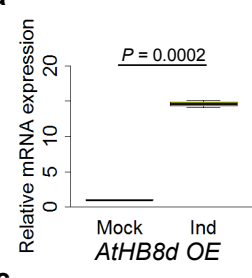

C

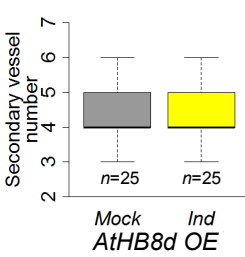

b

pANT:XVE $>A t H B 8 d-Y F P$

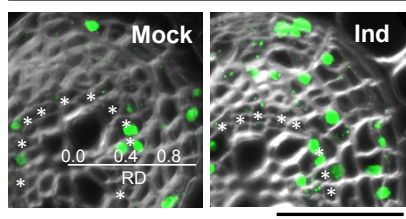

d
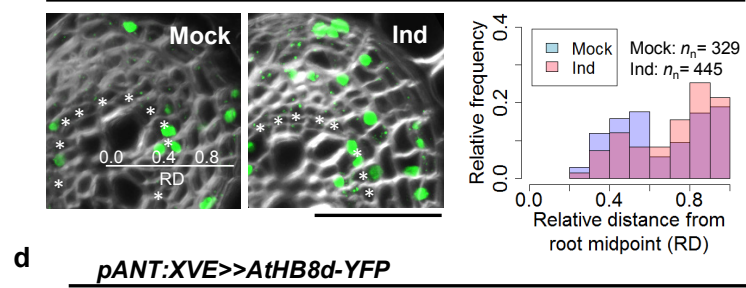

48h induction
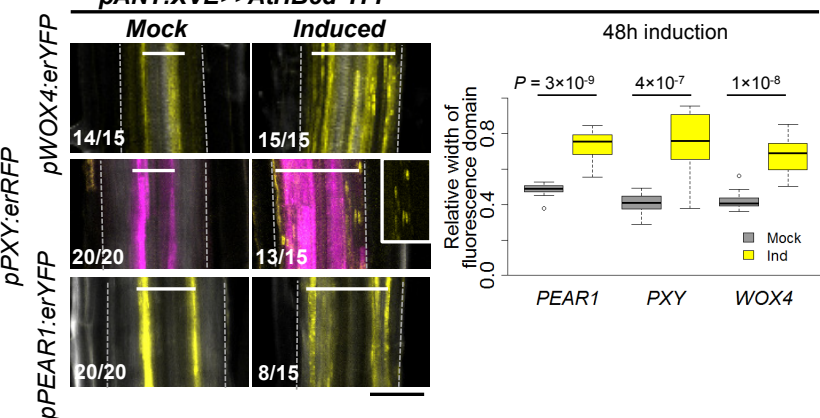

e
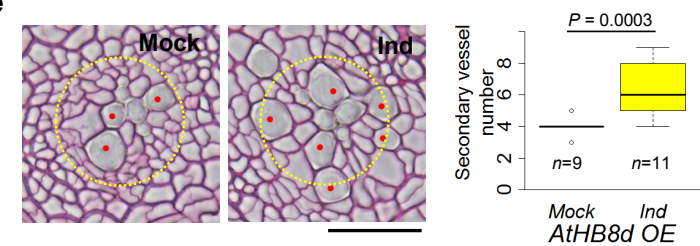

AtHB8d OE 


\section{Extended Data Figure 7}

a

b
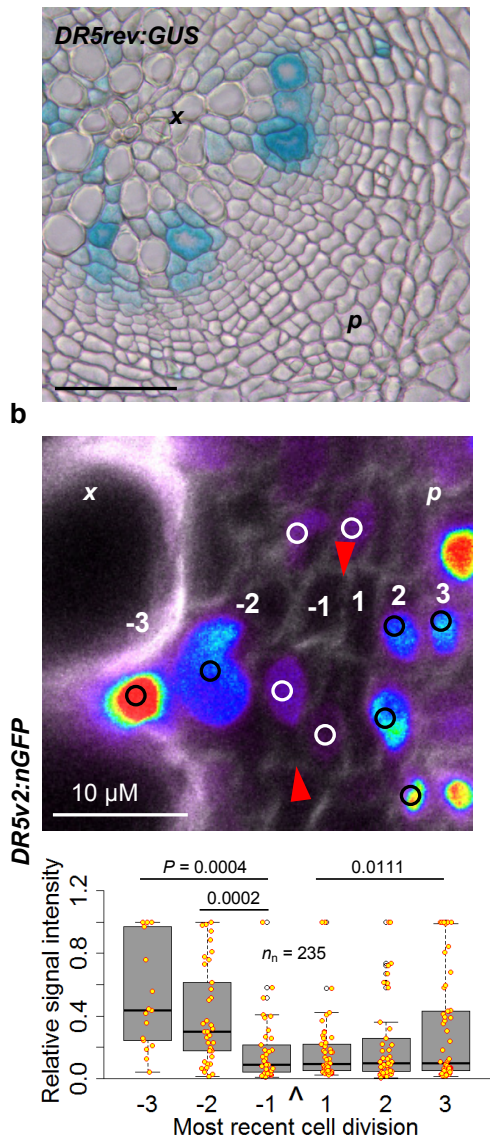

C

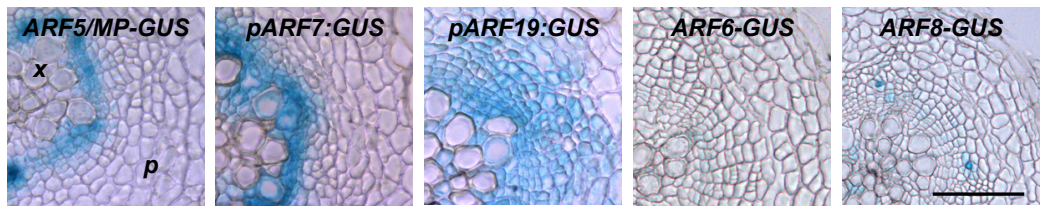

d
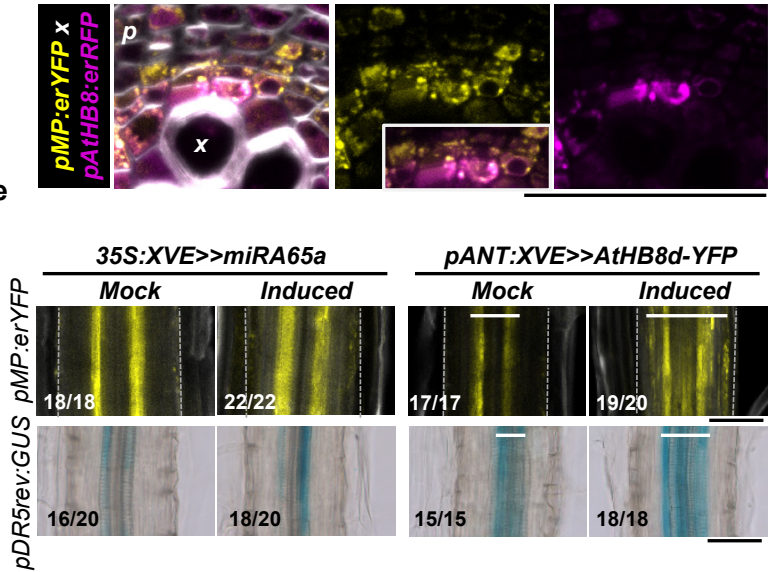

f

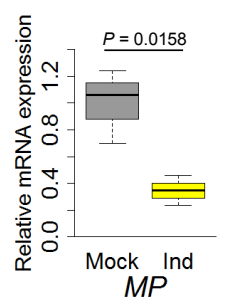




\section{Extended Data Figure 8}

a

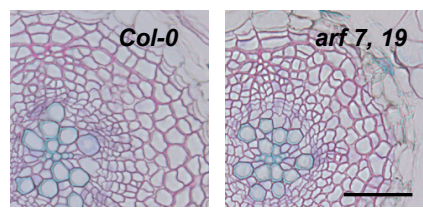

b

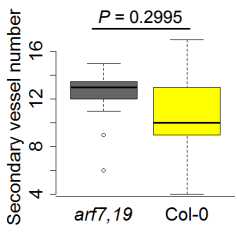

c

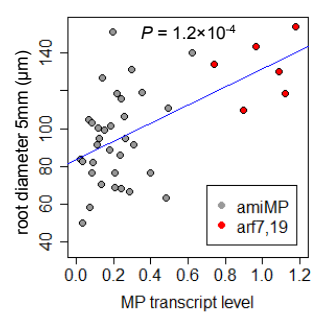

Analysed $5 \mathrm{~mm}$ below root-hypocotyl junction
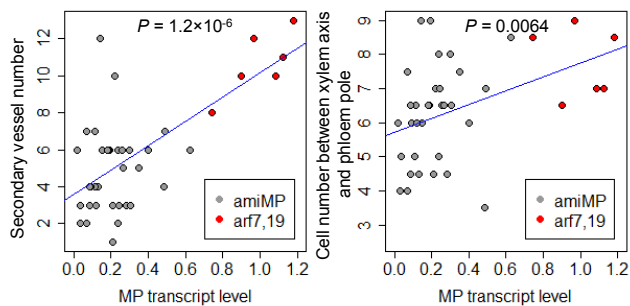

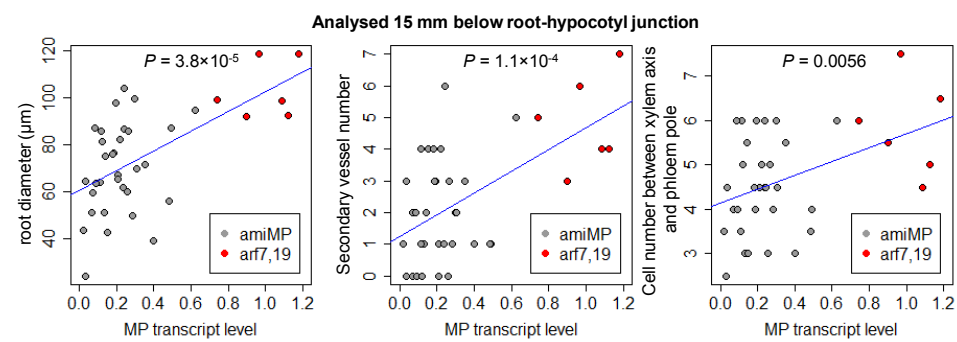

d pG1090:XVE>>axr3-1-RFP

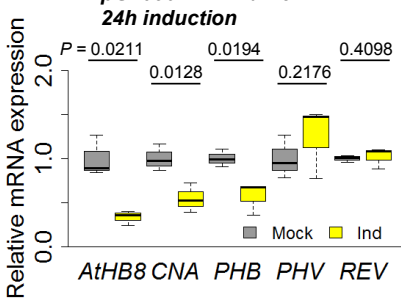

$72 h$ induction

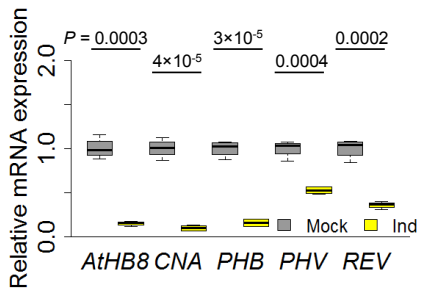

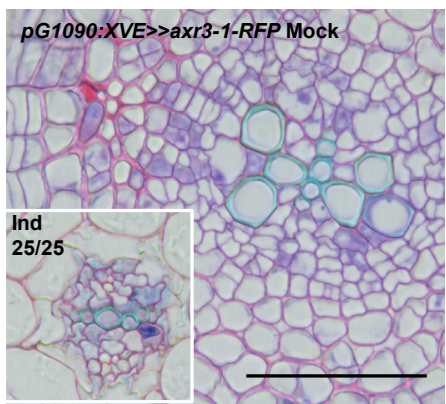

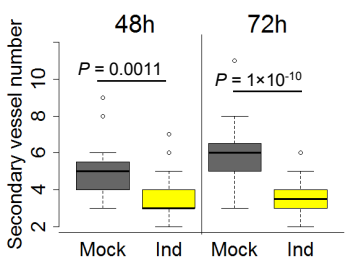




\section{Extended Data Figure 9}

a

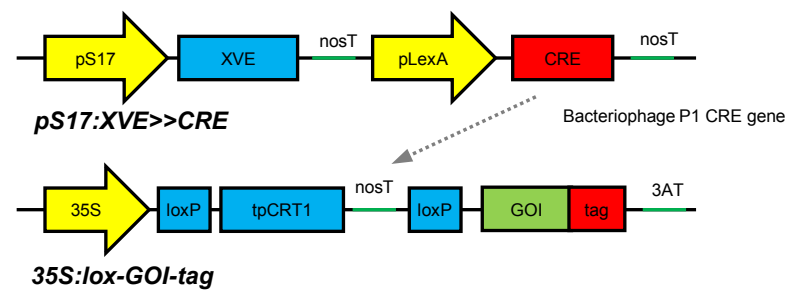

b

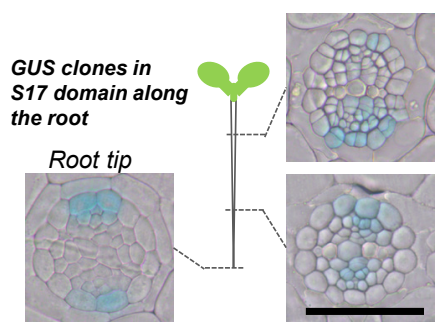

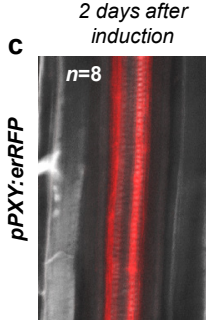
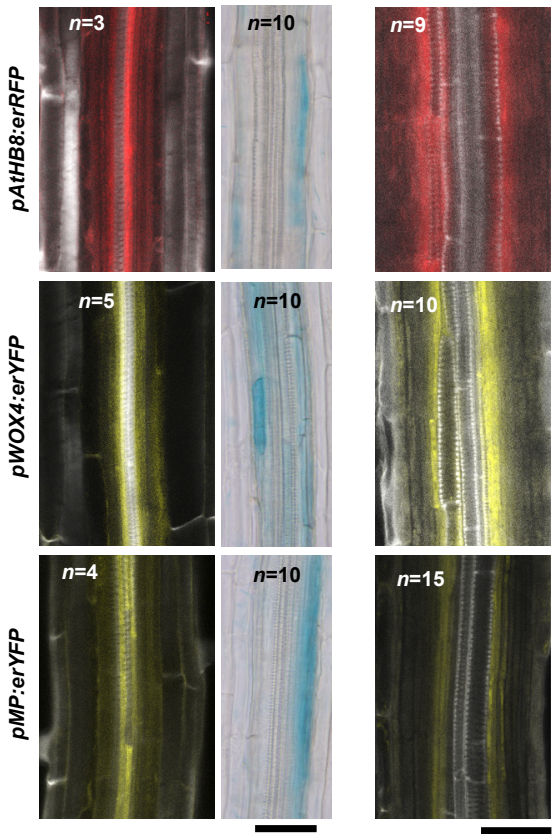

e
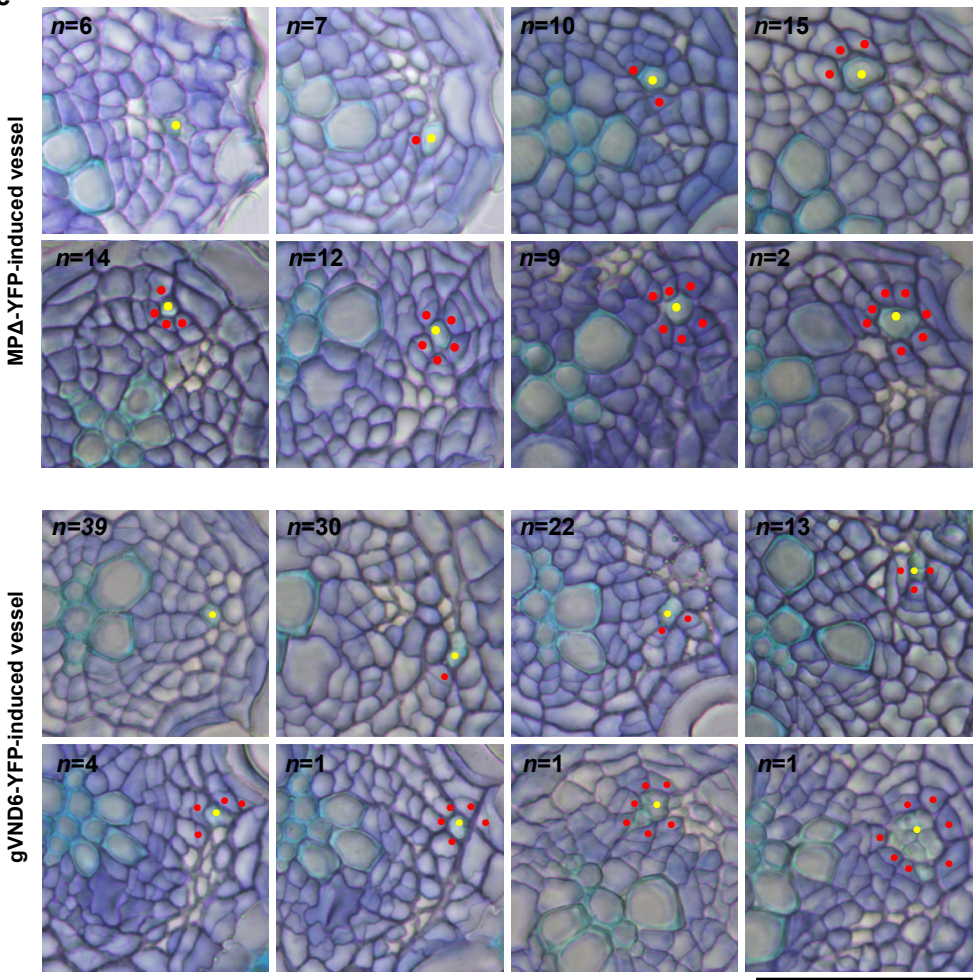

g

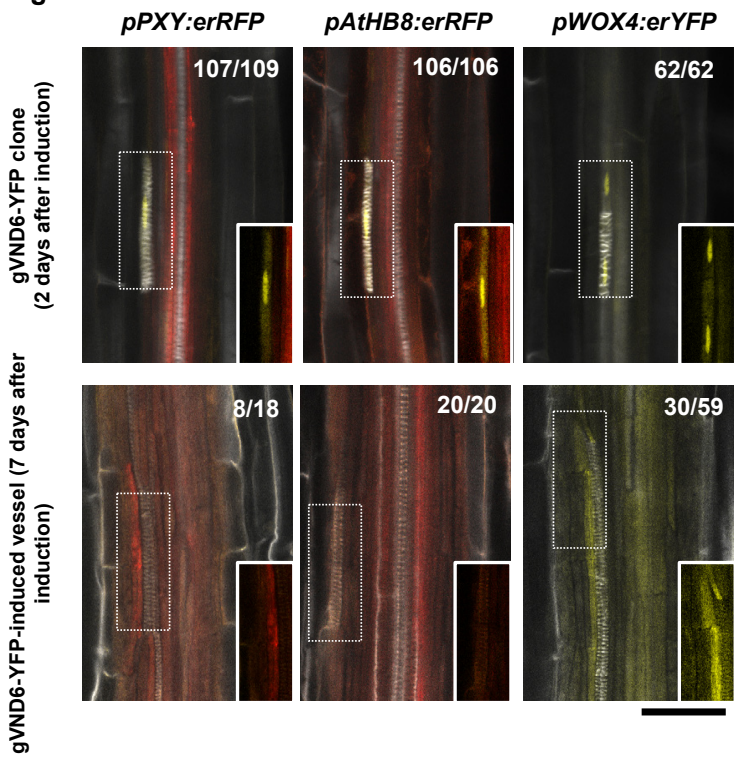




\section{Extended Data Figure 10}

a

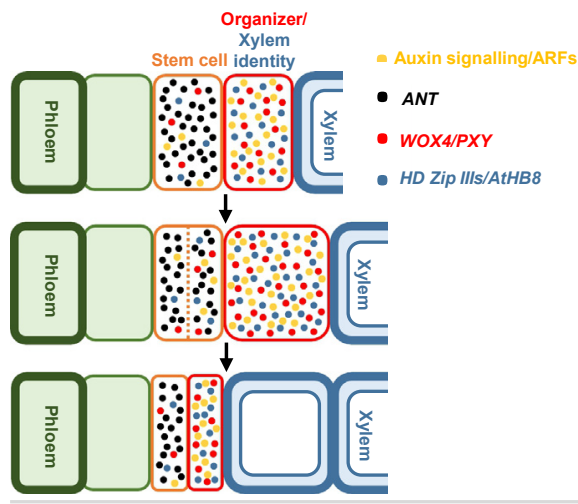

b

Vascular cambium

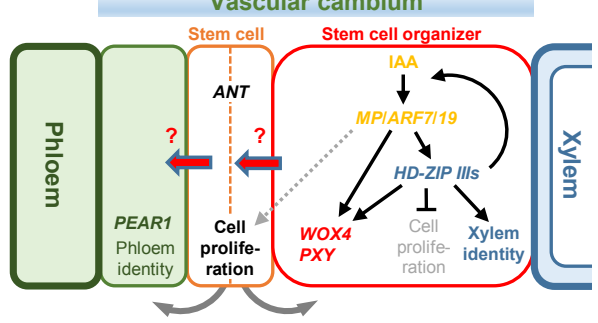

